\title{
The University of Wisconsin-Madison
}

\section{Torsatron/Stellarator Laboratory Program}

\author{
FY 1991-1993
}

\section{Annual Progress Report}

Grant DE-FĠ02-86ER53216.A010

\section{J.L. SHOHET, PRINCIPAL INVESTIGATOR}

\author{
D.T. ANDERSON, CO-PRINCIPAL INVESTIGATOR \\ F.S.B. ANDERSON, CO-PRINCIPAL INVESTIGATOR \\ J.N. TALMADGE, CO-PRINCIPAL INVESTIGATOR
}

\section{DISCLAIMER}

This report was prepared as an account of work sponsored by an agency of the United States Government. Neither the United States Government nor any agency thereof, nor any of their employees, makes any warranty, express or implied, or assumes any legal liability or responsibility for the accuracy, completeness, or usefulness of any information, apparatus, product, or process disclosed, or represents that its use would not infringe privately owned rights. Reference herein to any specific commercial product, process, or service by trade name, trademark. manufacturer, or otherwise does not necessarily constitute or imply its endorsement, recommendation, or favoring by the United States Government or any agency thereof. The views and opinions of euthors expressed herein do not necessarily state or reflect thos of tir United States Government or any agency thereof.

September, 1991

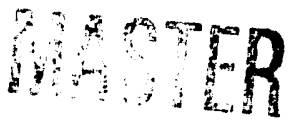




\section{Table of Contents}

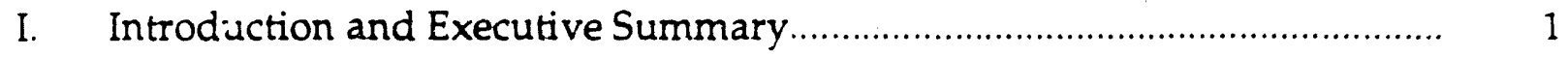

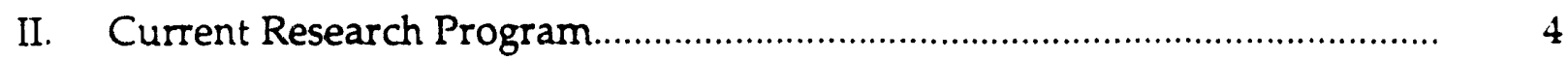
A. Fluctuations and Biasing Studies.

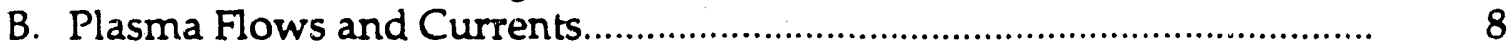
C. Quasi-Helically Symmetric Configurations.......................................... 11

III. Proposed Research Program............................................................ 14
A. Fluctuation-Induced Transport.
B. Plasma Currents and Flows in the Presence of Magnetic Islands.......... 15
C. Plasma Potential Structures and Direct-Orbit Loss with ECRH........... 16
D. Innovative Toroidal Systems Studies........................................................ 17
E. Projected Guals, Schedule, and Capital Equipment Request................ 19

IV. Proposed Budget 


\section{Introduction and Executive Summary}

This document summarizes results obtained during the first eight months of the current three year grant for research at the University of Wisconsin-Madison Torsatron/Stellarator Laboratory (TSL) and presents plans for future activity during fiscal years 1992 and 1993. Research efforts have focussed on fundamental physics issues associated with toroidal confinement, predominantly through experimental investigations on the Interchangeable Module Stellarator (IMS). The program direction has been guided into studies of fluctuations, potentials and electric fields, plasma currents and flows, and effects of magnetic islands by a desire for increased relevance and impact on the general toroidal confinement program. Theoretical and computational activities are also being undertaken to support the experimental research and to identify interesting new toroidal confinement concepts which could contribute to the understanding of tokamak transport.

Recent activities, in preparation for the second and third years of our present grant, are described in Section II. Briefly, our accomplishments during this grant period inclucle:

- Radial electric fields have been created in IMS through an electrode biasing technique. Emissive probe measurements, toroidally half-way around the torus from the biasing electrode, show field strengths up to $35 \mathrm{~V} / \mathrm{cm}$ with +80 volts applied bias.

- Sheared poloida! flow velocity distributions have been measured directly by two methods (fluctuation phase delay and ion flow) in the region of the impressed radial electric field. Results from the two methods are in good agreement with each other and with $E \times B$ velocity estimates. $\left(V_{\text {flow }} \sim 14 \mathrm{~km} / \mathrm{s}\right)$

- The line-averaged density can be increased with bias. Additionally, the electron density profile can be changed from hollow to nearly flat with the application of bias depending on the resonance location. Fluctuation amplitudes have been reduced in a region surrounding the biasing position.

- A novel probe has been developed and tested to simultaneously measure three components of local fluctuating plasma electric fields and densities in preparation for studying the detailed effects of poloidal flows on fluctuations and measurement of the Reynolds stress tensor. 
- A code has been developed capable of calculating the Pfirsch-Schlüter currents in the presence of magnetic islands.

- A paddle-probe has been constructed and tested for measurement of "smale-scale" plasma currents. Initial measurements indicate qualitative agreement with calculations not including the error fields. The probe, by examining only the ion flow, was also used to look at poloidal flow measurements, discussed above, showing good agreement with other diagnostics.

- An error field which can be used for the computation of magnetic fields in IMS has been developed which gives good agreement with experiment on phase and size of the magnetic islands. The model error field will be used in future current calculations and to guide later removal of the islands.

- Laboratory developments have included: test operation of a new, higher power, microwave source; improved flat-top of the magnet pulse (<.1\% for $14 \mathrm{~ms}$ ); extensive upgrading of the data acquisition system; demonstration of RF plasma production in Proto-Cleo with densities $\left(2.5 \times 10^{12} \mathrm{~cm}^{-3}\right)$, an order of magnitude higher than that using the present ECRH system.

- Quasi-Helically symmetric (QHS) con'igurations, with nearly perfect collisionless.single particle confinement, have been identified at aspect ratios as low as 6 .

- Monte-Carlo calculations demonstrate a factor of three reduction in neoclassical diffusion losses for the QHS configuration compared to an equivalent tokamak at all collisionalities.

The accomplishments during the present grant period have positioned the Torsatron/Stellarator Laboratory to investigate important issues in toroidal confinement. These issues and plans are discussed in Section III. Specificaily, our proposed activities for the next two fiscal years include:

- Measurement of radial electric field strength and distribution as a function of applied bias voltage and current. Determine the relationship between flow velocity and radial electric field strength.

- Measure details of potential and density fluctuation levels, correlations and fluctuation-induced transport with different levels of biasing (and thcrefore poloidal flow velocity and velocity shear).

- Calculate the poloidal viscosity by varying the bias voltage and observing the spin-up time of the plasma. 
- Measure $\partial / \partial \mathrm{r}\left\langle\mathrm{V}_{\mathrm{r}} \mathrm{V}_{\theta}>\right.$ to calculate the poloidal flow component from the Reynolds stress tensor and compare to observed poloidal flow measurements for comparison to the theory of Diamond and Kim.

- Determine the effects of poloidal flows on potential and density asymmetries associated with magnetic islands.

- Measure planar distribution of Pfirsch-Schlüter currents in the vicinity of the already-mapped islands in IMS.

- Use an external dipole coil to experimentally confirm the source of the error fielit present in IMS. Investigate ability to control island size with this external coil. Controlled islands will be used to augment the asymmetry/flow studies mentioned above.

- Take IMS down for repair of the field error and installation of discriminator screens on the divertor strike-plates for direct-loss studies. Re-examine asymmetry studies in the absence of vacuum islands.

- Investigate effects of direct orbit loss on confinement and plasma potential structures through variation of the resonance location and magnetic topology. Measure energies and spatial distributions of lost particles using gridded energy analyzers (GEA's) in the divertor regions.

- Continue exploration of innovative toroidal concepts which could have a significant experimental impact on the understanding of toroidal confinement, with emphasis on quasi-helically and quasi-axially symmetric configurations of the HELIAS family.

Also included in Section III is a list of our projected goals and schedule and capital equipment needed to carry out the proposed program. 


\section{Current Research Program}

The research efforts in the $T$. satron/Stellarator Laboratory at the University of Wisconsin-Madison during the present grant period have been tailored to increase the impact of our research in the support of mainline concepts. For example, edge fluctuations and poloidal rotation play a central role in toroidal confinement and appear intimately linked to observed $\mathrm{L}-\mathrm{H}$ mode transitions observed in tokamaks. As a result, Section II.A reports on the work we have done to date on fluctuation-induced transport and the effects of poloidal rotation induced in IMS through a biasing electrode. Theoretical models developed by Boozer, Reiman, and more recently Kadomtsev, suggest plasma currents can interact with magnetic island structures in the plasma to degrade confinement. Two-dimensional current distributions (with and without the presence of islands) have not been measured as yet in any toroidal device. At present, we are making such measurements in IMS for comparison to theory. Additionally, poloidal asymmetries which are observed in CCT (and are coincident with rational rotational transform locations) disappear with the onset of poloidal rotation. The detailed knowledge of the structure of the $q=3$ island in IMS is being used to investigate the issues of currents and asymmetries. Section II.B discusses preliminary results and the work done to date in preparation for rnore detailed studies. Finally, innovative new toroidal configurations have been identified which could offer an attractive test facility for examining the possibility of improving confinement over that of present tokamaks and stellarators. Work in this area is presented in Section II.C.

\section{A. Fluctuations and Biasing Studies}

Current studies at TSL in the area of fluctuation-indl'ced transport are designed to address key issues in (letermining the role of this plasma loss mechanism in toroidal confinement devices. Of particular interest is the area of the $L$ to $H$ confinement mode transitions seen in many tokamaks. Reports on these transitions indicate that a sheared edge-plasma rotation velocity accompanies the transition to the H-mode, either spontaneously in DIII-D 1 , or induced in CCT ${ }^{2}$ or TEXTOR ${ }^{3}$. One of the ctntral issues is the correlation between this sheared rotational flow and the reported reduction of the electrostatic fluctuation-induced transport. Theories have suggested that the shearing effect of the velocity caused the reduction of the fluctuation transport 4 . Numerical models implemented to study the effect of a sheared velocity profile on the non-linear saturation levels of the turbulence, however, show the saturated level to be unaffected by the sheared velocity profile 5 . Results from the TEXT device, presented by Ritz at the Madison H-Mode Workshop (August, 1991) still seem to support this theory by observing reduced fluctustion levels with a sheared poloidal velocity. The fluctuations themselves may cause the plasma edge rotation through the Reynolds stress 6 , but this has yet to be examined by experiment and will be addressed in our future research. 
It is apparent from this rapidly-changing area of study that detailed measurements of the fluctuation-induced transport in the presence of sheared plasma flow is needed to help substantiate theoretical models and guide the physical understanding of these processes. IMS offers a good experimental platform to study this subject because, 1) its plasma parameters allow the use of Langmuir probes to acquire the necessary, detailed, local measurements of density, temperature and plasma potential fluctuations, 2) its magnetic geometry is known very accurately, and 3) although an $\mathrm{H}$-mode has not been generated in IMS, a large radial electric field can be induced by a positively-biased electrode placed just inside the separatrix. This radial field has been observed to drive a poloidal rotation of the edge plasma. The remainder of this section documents the present fluctuation studies by examining: the experimental setup and techniques in which the measurements are performed, the plasma parameters during normal and "biased" modes, and the experimental evidence for plasma poloidal rotation. Finally, the influence of the induced radial electric fields/poloidal rotation on the fluctuating parameters is presented.

\section{Experimental Setup for Fluctuation Measurements}

The diagnostic used in measuring the fluctuating plasma parameters is shown in Figure II.A.1. There are six probes concentrated in a small space on the end of ceramic (alumina) shafts connected to a positioner assembly which allows the array of probes to scan the entirc poloidal cross section of the plasma. Two of the probes are simple Langmuir probes in which one is biased into ion-saturation and used to calculate the plasma density and its fluctuations. The other probe is referenced to ground through a high resistance (10 M $\Omega$ ) which allows it to follow the plasma floating potential. The value of the floating potential is then compared to the measured plasma potential to infer an electron temperature. The other four probes are emissive probes which consist of a 1 mil diameter tungstien wire. These probes are arranged to form a basis set of three vectors to obtain a three dimensional representation of the plasma potential distribution. This arrangement is necessary to resolve the spatial distribution of the fluctuations into the proper components that drive the plasma flux normal to, and tangent to the magnetic flux surfaces. Since the IMS magnetics are well known, including the locations of the islands, it is possible to observe the effects of these islands on the fluctuation-induced transport characteristics as well.

The IMS edge plasma rotation is accomplished by the insertion of a large positively biased electrode into the plasma located $1 \mathrm{~cm}$ inside the separatrix as shown in Figure II.A.2. The electrode is elongated in shape and oriented tangent to the magnetic flux surface. The biasing potential used for the fluctuation experiments was $+80 \mathrm{~V}$ which is sufficient to reduce the electron population on the magnetic flux surface, and cause the plasma to reference its potential locally to that of the probe. The current required to maintain the probe voltage at $+80 \mathrm{~V}$ is approximately $0.45 \mathrm{~A}$ which is substantially higher than the $0.1 \mathrm{~A}$ total current flow 


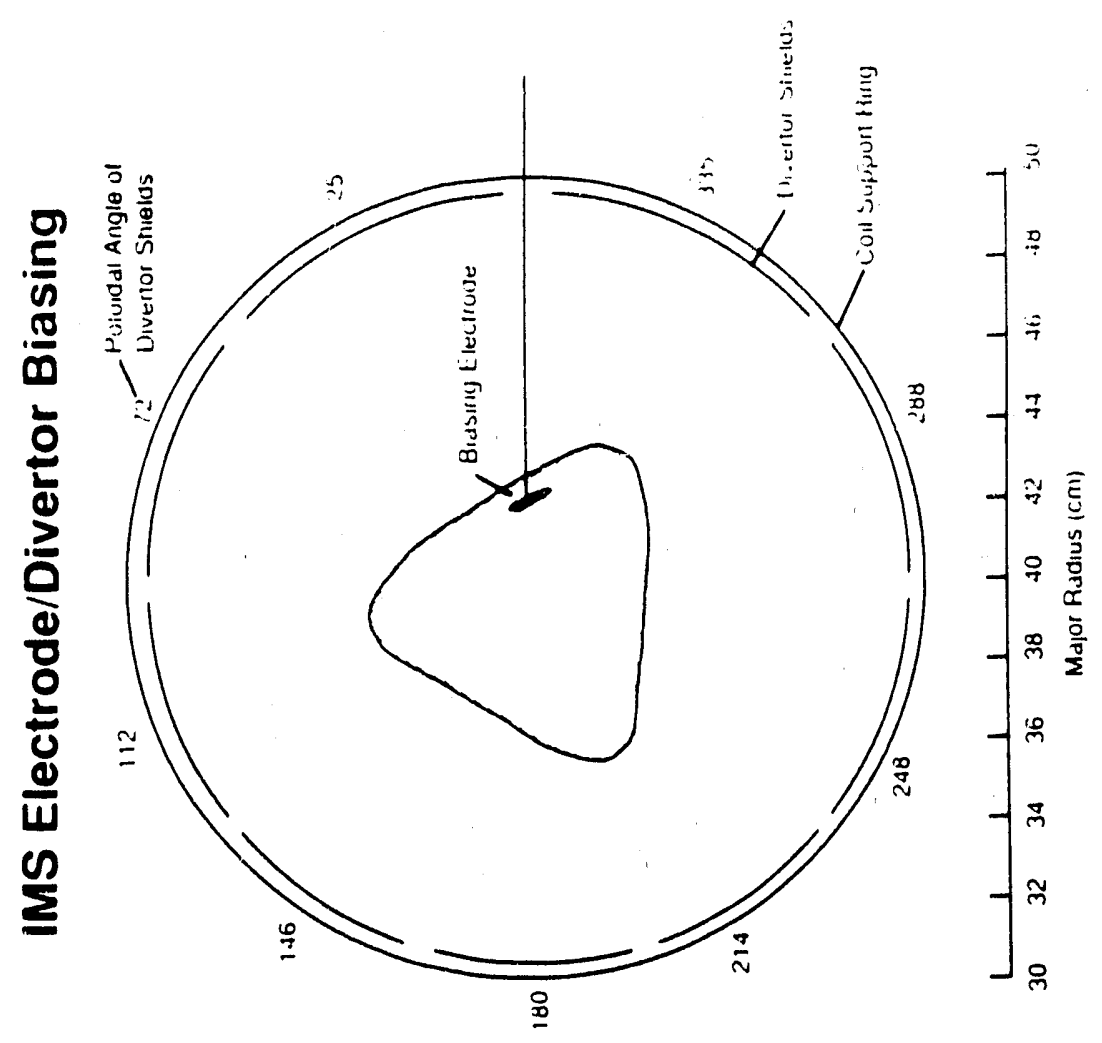

$\stackrel{1}{3}$

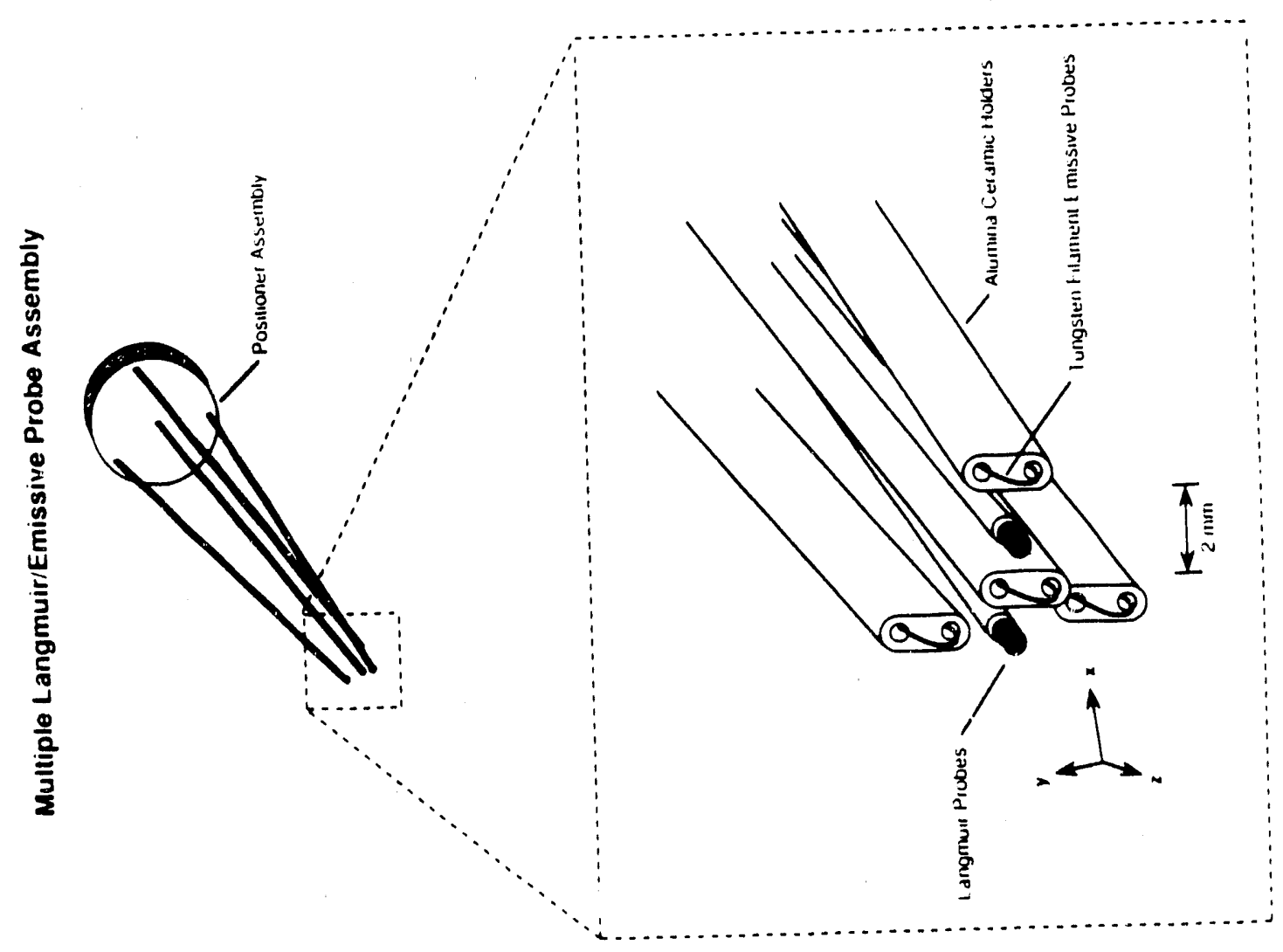

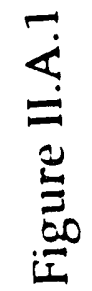


out of IMS during a normal discharge. Yet, the plasma density, as measured by the microwave interferometer, increases by approximately $20 \%$ in the measurements reported here. The application of the $+80 \mathrm{~V}$ bias on the electrode is done during half of the $10 \mathrm{~ms}$ IMS discharge, otherwise, the electrode is electrically disconnected. Thus, the impact of electrode biasing can be seen within a single plasma discharge. This method will allow a study of the important dynamics in the transition from one plasma rotation state to another.

\section{Plasma Parameters During Electrode Biasing and Plasma Rotation}

The most dramatic effect of the electrode biasing is seen in the plasma potential profile shown in Figure II.A.3. For the experiments reported here, the ECH resonance was located near the axis of the plasma. The separatrix is located at the major radius values of $35.5 \mathrm{~cm}$ and $42.5 \mathrm{~cm}$ for this scan, and the electrode is positioned at $41.5 \mathrm{~cm}$. The lower trace is for the case of no applied bias to the electrode, and is seen to be almost flat across the cross-section of the plasma. The upper trace shows the potential structure when the electrode is biased to $+80 \mathrm{~V}$, and reveals the strong potential gradients introduced toward the edge regions. The upper trace in Figure II.A.4 shows the corresponding plasma density profiles. The error bars in the figure reflect the local density fluctuation levels. The hollow density distribution remains during the electrode biasing, but the density peaks are higher and oriented closer to the magnetic axis of IMS $(39.0 \mathrm{~cm})$ with an approximate $20 \%$ increase in line-averaged density. With the ECRH layer located near the inboard side of the device, the density profile changes from hollow to nearly peaked as shown in the lower plot in Figure II.A.4 (a subject of future investigation). The electrode biasing has no large effect on the electron temperature profile.

The gradients in the potential profile of Figure II.A.3 iridicate a strong, minorradially directed, positive electric field which gives rise to an $E \times B$ drift in the poloidal direction. Figure II.A.5 shows the calculation of this velocity for the $+80 \mathrm{~V}$ bias case. The velocity plotted is in the poloidal direction so that velocities of the same sign on opposite sides of the plasma are indicative of poloidal rotation. This graph, therefore, indicates there should be a strong poloidal rotation of the edge plasma in the vicinity of the biased electrode. Two s\&parate measurements were made to verify this plasma rotation.

The first method used an array of four ion-saturation probes aligned so that they are located on the same magnetic flux surface in the poloidal dirtetion. Assuming the ion-saturation piobes reflect thi plasma density fluctuation characteristics, one can compare the signal from each of the probes in frequency space to see if they are well correlated, and if so, if there is a linear phase shift vs. frequency of the individual components. Calculating the slope of the phase relationship gives the time delay and, with the known distance between the probes, allows the calculation of the flow velocity. Figure II.A.6 shows the results of these calculations which show good agreement with the calculations of Figure II.A.5, with the exception that the velocity distribution extends over a wider range of major radius. Data is not presented for the interior regions of the plasma as phase shifts there were too small compared to the minimum digitizer sampling interval. 


\section{Plasma Potential Profile}

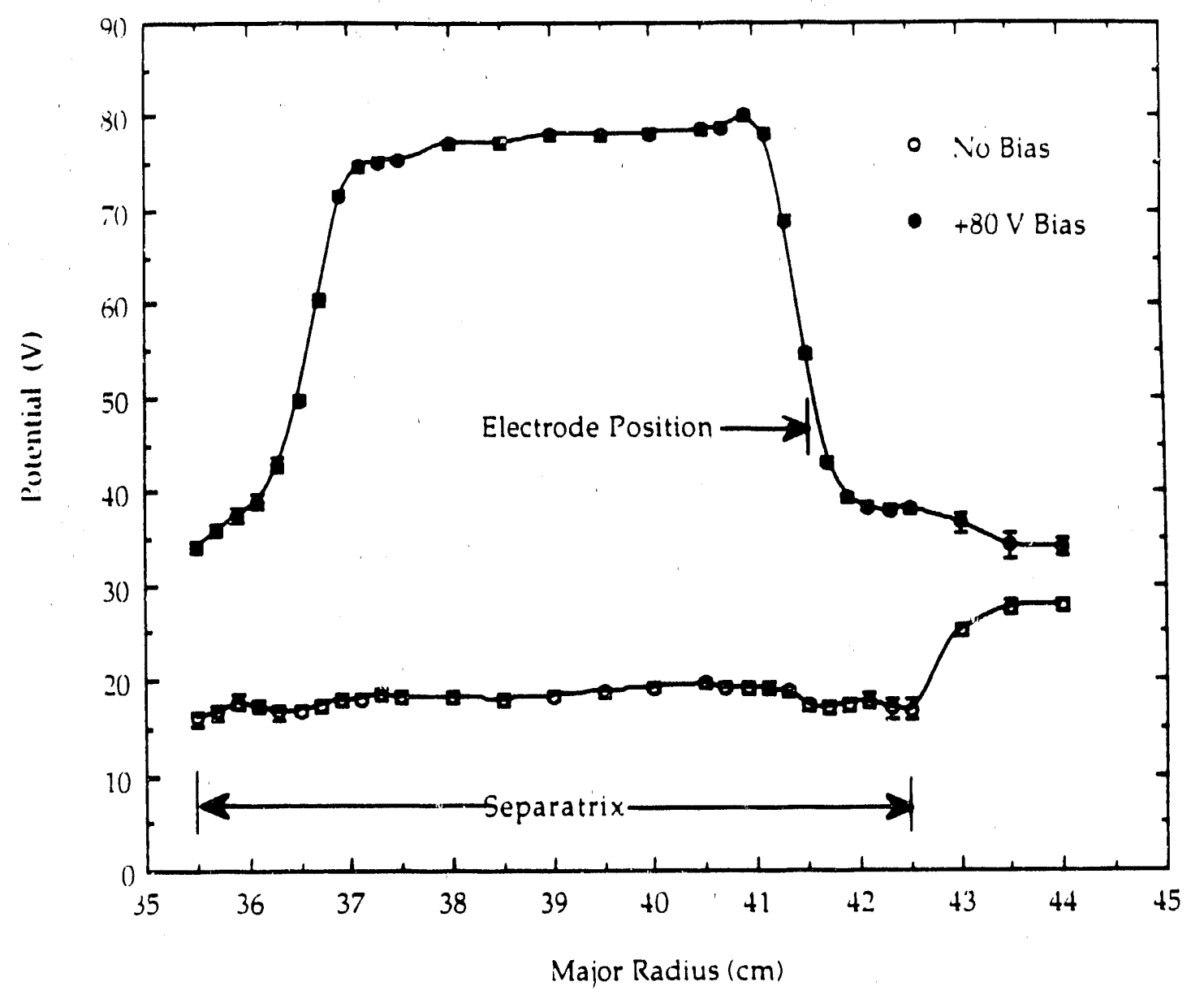

Figure II.A.3 
Plasma Density Profile 6000 A

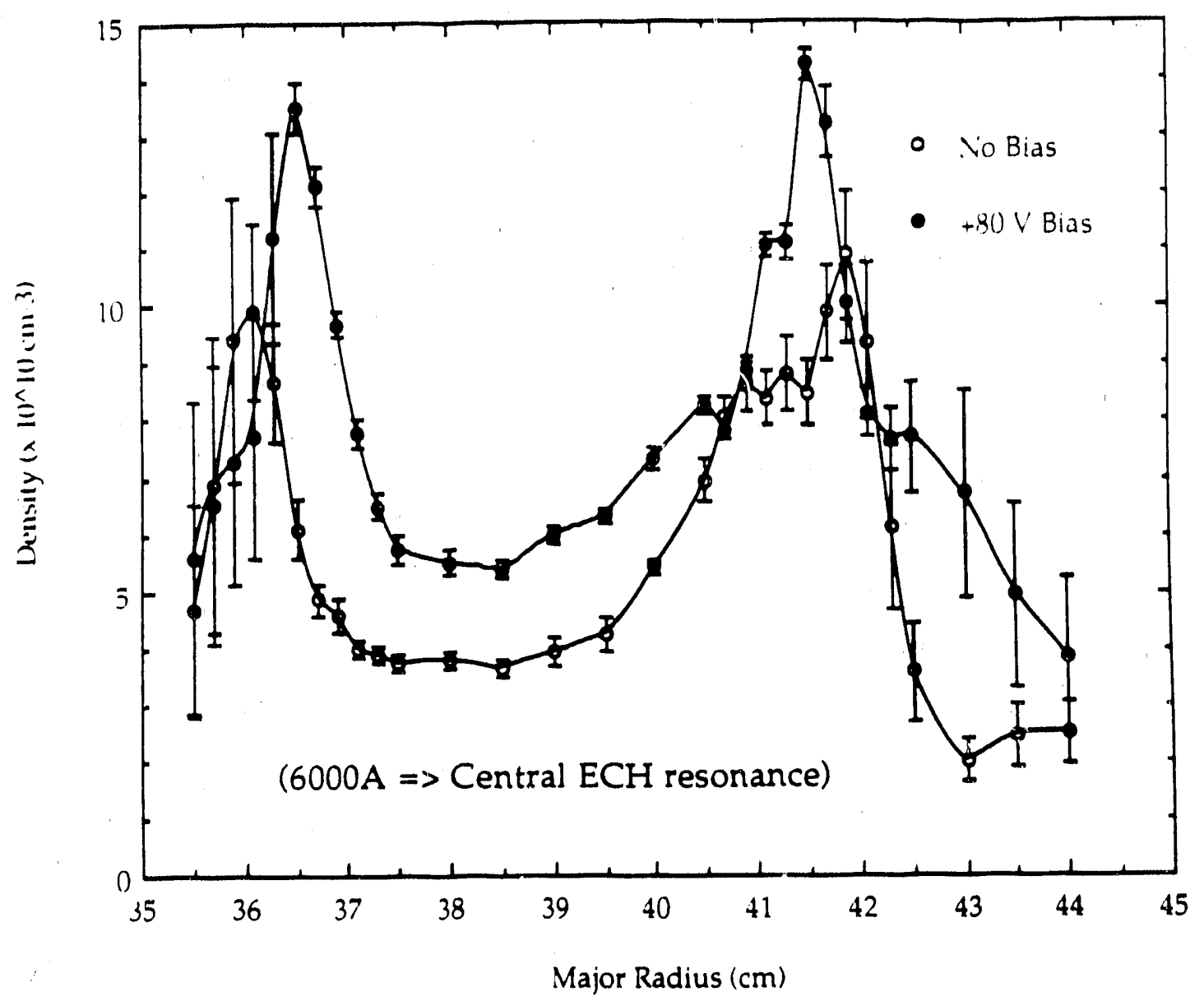

P'lasma Density l'roflle, $5200 \mathrm{~A}$

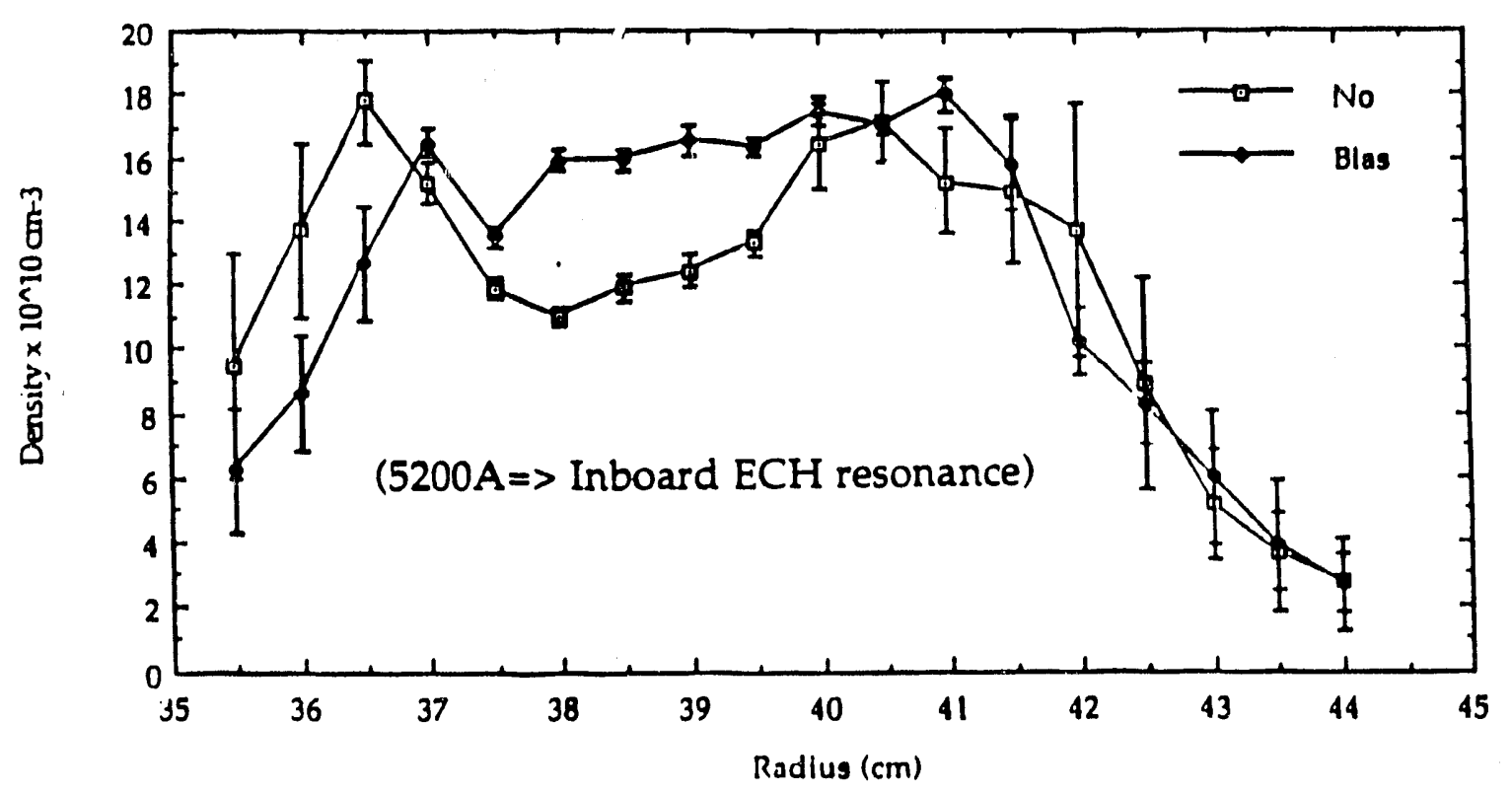

Figure II.A.4 
'Poloidal E $\times$ B Velocity Protile During Electrode Bias

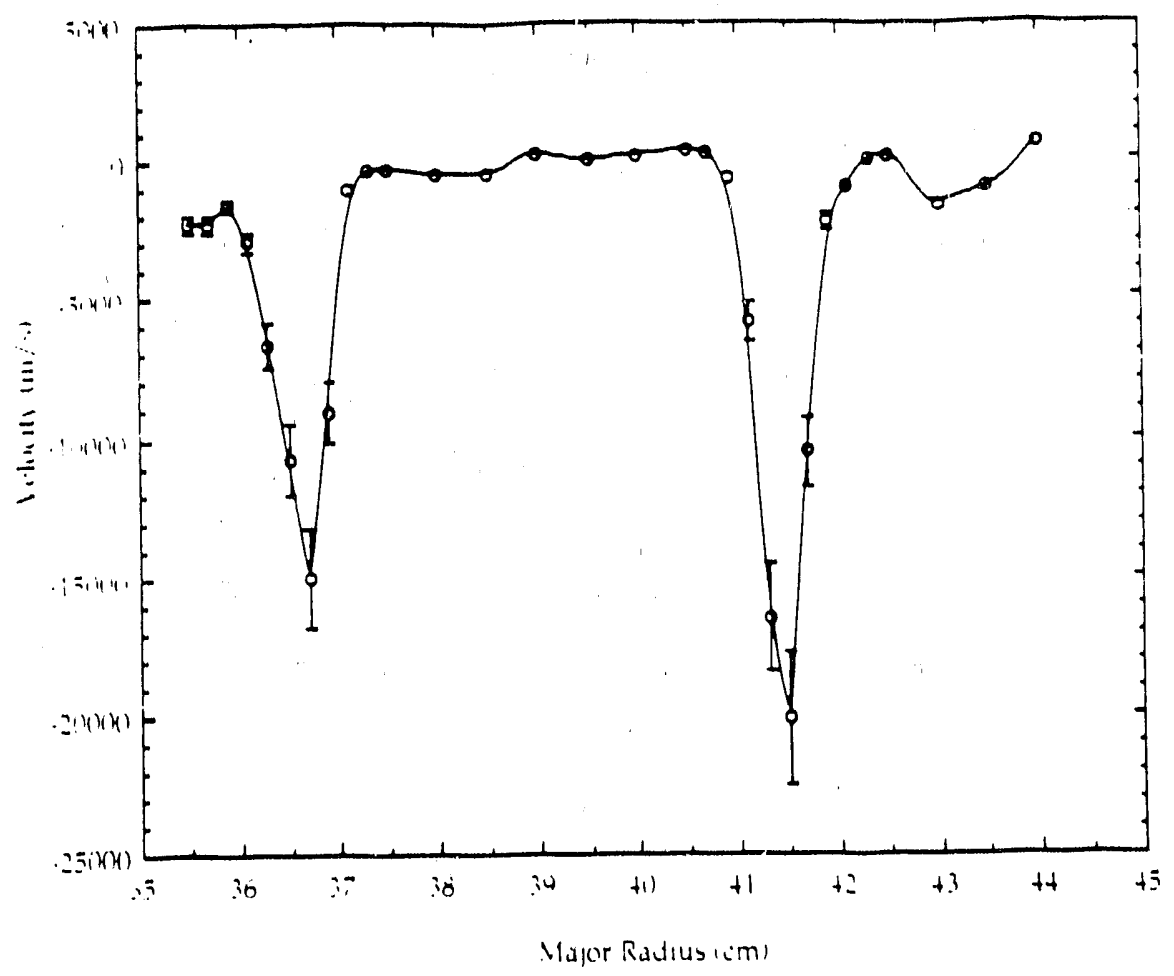

Figure II.A.5

Poloidal Velocity From Phase Delay During Electrode Bias

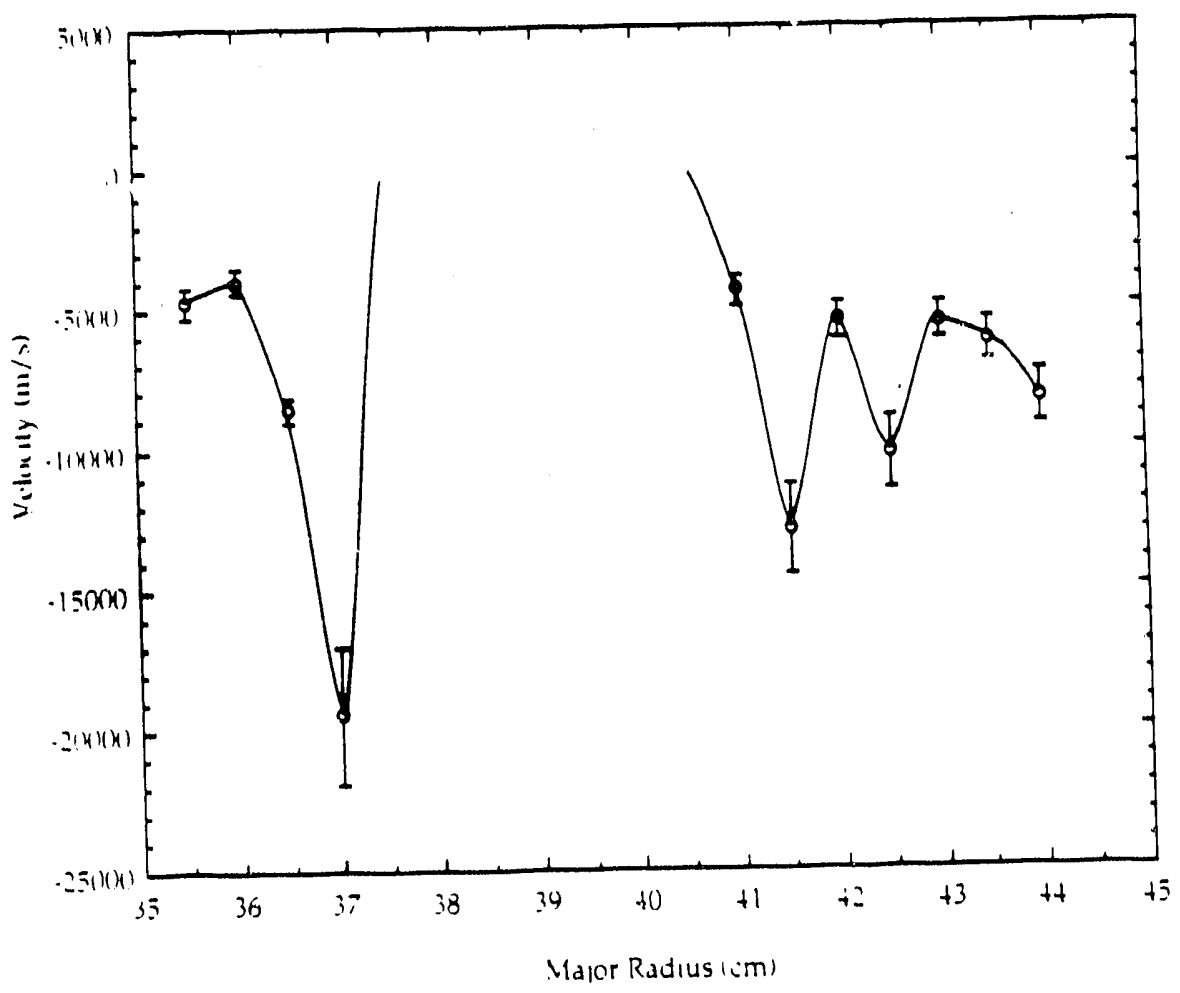

Figure II.A.6 
The second method utilizes the scheme currently implemented to measure the Pfirsch-Schlüter currents in IMS. This technique is described in Section II.B of this report. The measurement probe was turned 90 degrees to the toroidal direction so that it measures the poloidally directed current. Preliminary results are shown in Figure II.A.7. This figure shows the poloidal ion current distribution. The corresponding electron flow is in the same direction as the ion flow with the direction of the flow in the $\mathrm{E} \times \mathrm{B}$ direction. This measurement shows the narrow velocity distribution consistert with that shown in Figure II.A.5. The peak current density $(j=n q V)$ yields an io in velocity of $-10,000 \mathrm{~m} / \mathrm{s}$, also in reasonable agreement with Figure II.A.5.

\section{Effects of Electrode Biasing on Fluctuations}

With the confirmation of the poloidal edge plasma rotation, the effect of this rotation on the fluctuation characteristics becomes of interest in light of the theoretical developments referred to earlier. Examination of the normalized density fluctuation profile in Figure II.A.8 shows reduced density fluctuation levels in the shear layer, but increased fluctuations elsewhere. Large reductions $(\sim 50 \%)$ are observed in the region from $40.5-42.5 \mathrm{~cm}$ on the outboard side of the device, a region slightly larger than the measured poloidal rotation layer. The fluctuation levels of the plasma potential with and without biasing are snown in Figure II.A.9. The plasma potential fluctuations, in the regions of plasma rotation, show an increase on the inboard side of IMS, while showing a decrease on the outboard side. The implications of these observations is not completely clear as far as the fluctuation-induced particle flux is concerned. The calculation of the transport for these discharges is still in progress. However, the fact that these parameters are influenced by the plasma rotation is important and warrants careful study in the future.

\section{References for Section II.A}

[1] R. J. Groebner, et. al., Phys. Rev. Lett. 64, 3015 (1990).

[2] R. J. Taylor, et. al., Phys Rev. Lett. 63, 2365 (1989).

[3] R. Weynants, et. al., in Proc. of the 17th European Conference on Controlled Fusion and Plasma Physics, Amsterciom, The Netherlands, June 25-29, 1990.

[4] H. Biglari, P. H. Diamond, and P. W. Terry, Phys Fluids B 2, 1 (1989).

[5] B. Carreras, riesented at Madison H-Mode Workshop, August 1991.

[6] P. H. Diamond and Y. B. Kim, Phys. Fluids B 3, 1626 (1991). 
Z-Directed Ion Current from Paddle-probe During Electroc.e Biasing

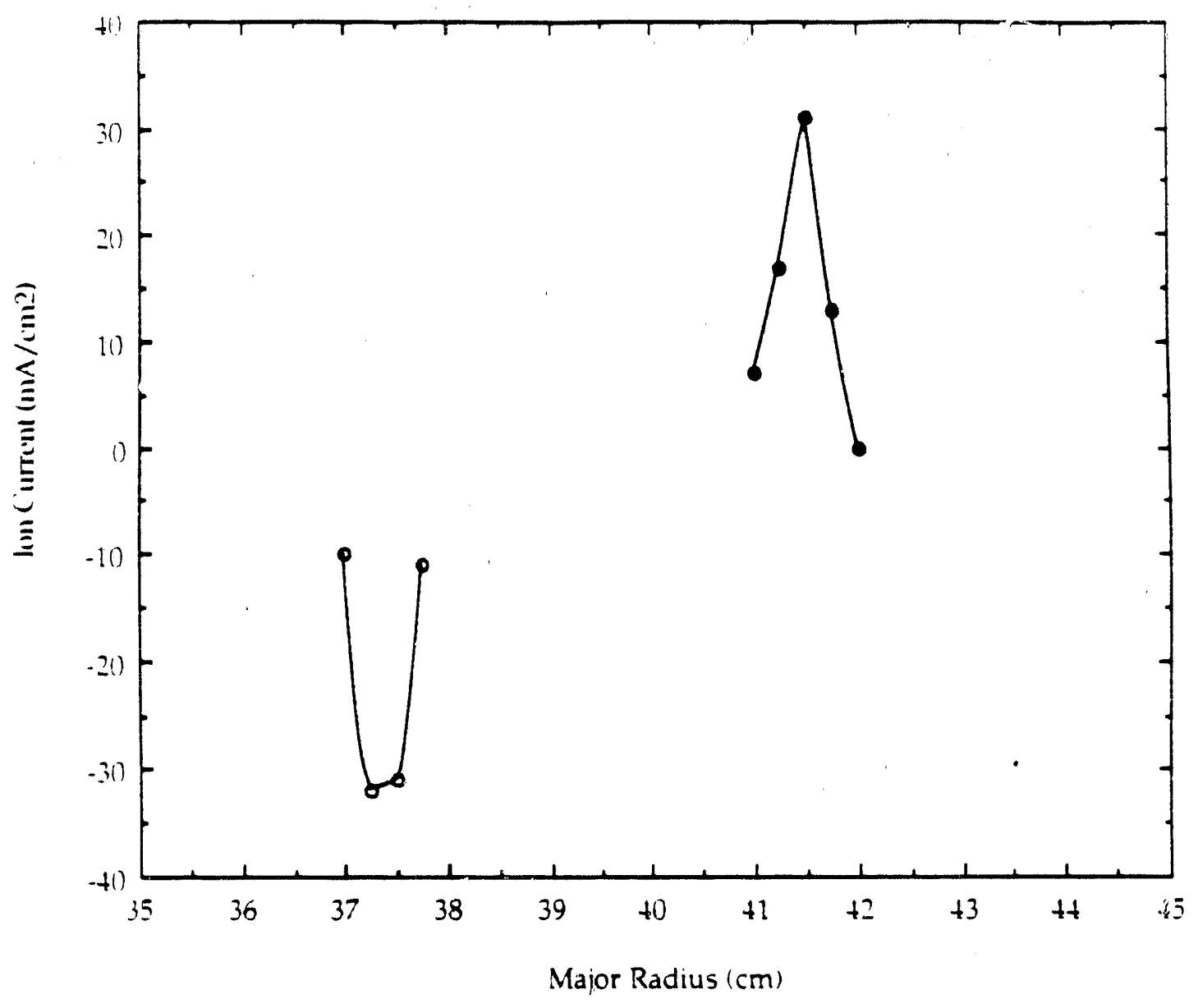

Figure II.A.7 
Plasma Density Fluctuation Profile

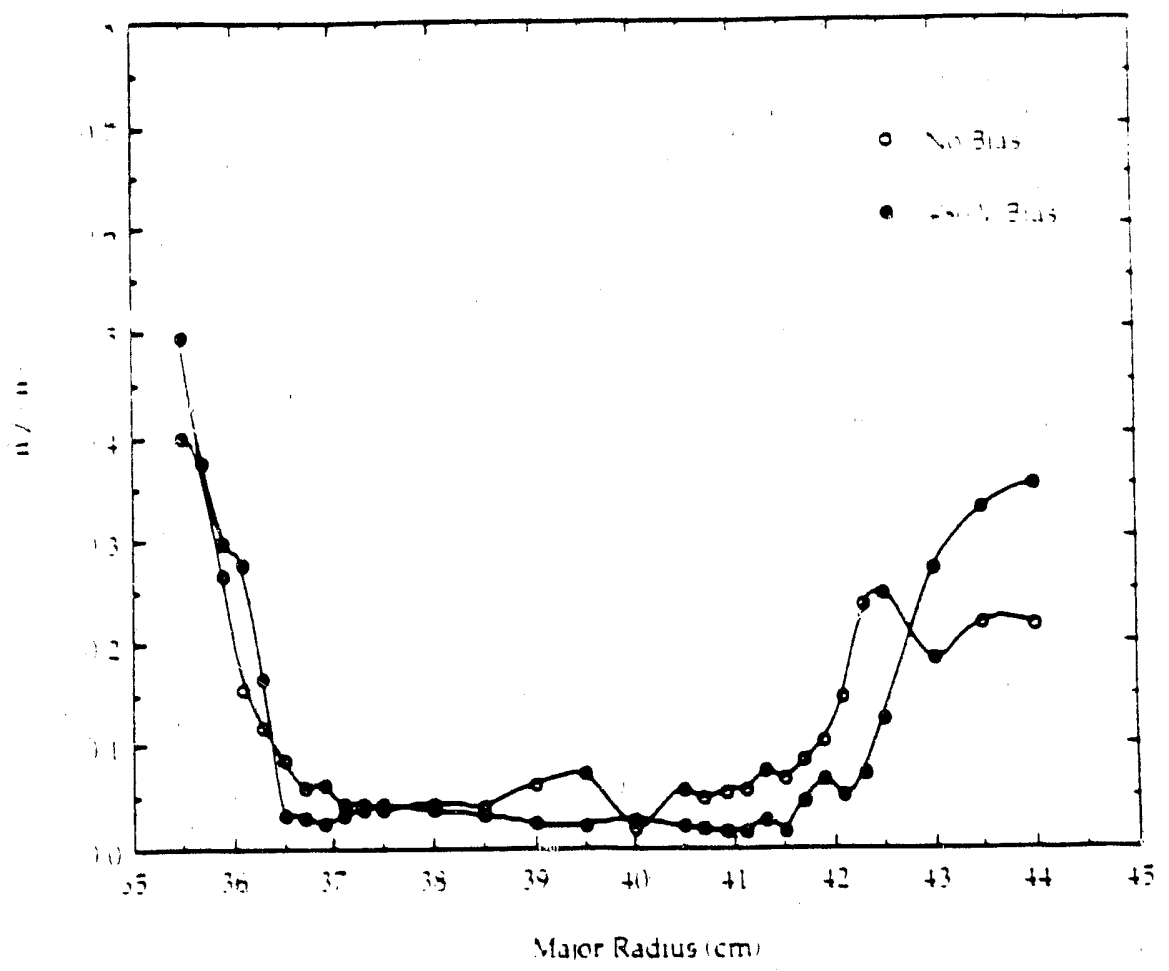

Figure II.A.8

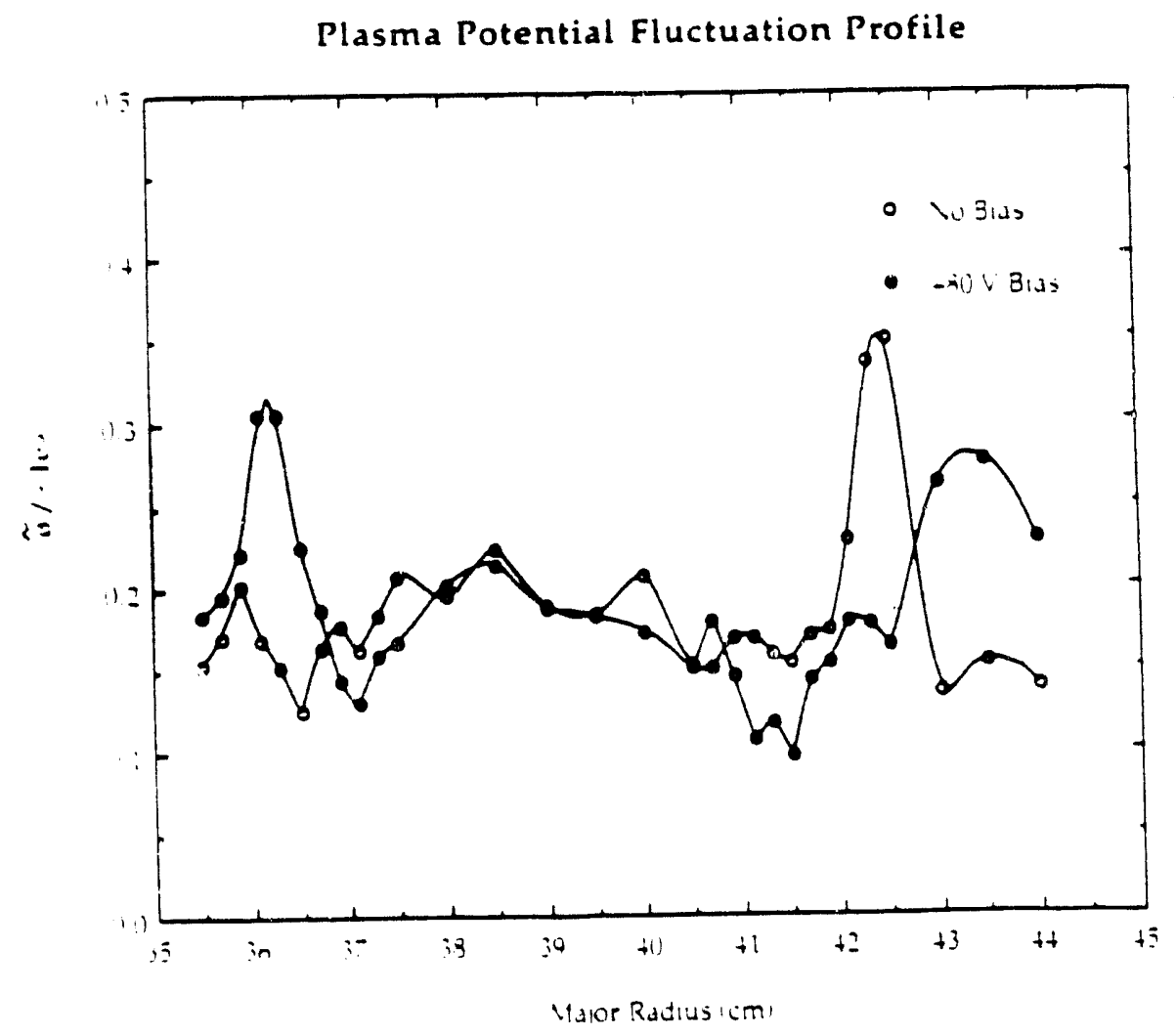

Figure ỉi.A. 


\section{B. Plasma Flows and Currents}

An important issuc 'or tokamaks and stellarators is the formation of magnetic islands and their resulting effect on transport and confinement. The threat that such islands pose to toroidal confinement lies in their ability to grow with plasma pressure from nonexistent or finite vacuum islands to the point where they overlap and destroy or degrade the nested magnetic flux s'urfaces necessary for good confinement ${ }^{1}$. This phenomenon can place a limit on beta for toroidal devices which often is lower than that of the Shafranov shift ${ }^{2}$. Field mapping and detailed measurements of current profiles in tokamaks have not been possible due to the presence of large ohmi: currents. These currents will be measured in the vicinity of well-mapped magnetic islancis in IMS, and compared with numerical calculations.

In addition, IMS offers the possibility of studying the effects of a controlled radial electric field on observed poloidal prcssure asymmetries. $M=3$ poloidal pressure asymmetries have been measured in INS, which correspond to vacuum magnetic islands inapped at the $q=3$ surface. In the CCT tokamak similar $m=3$ poloidal asymmetries were also observed at the $q=3$ surface 3 . The poloidal asymmetries in CCT were removed by using a radial electric field to induce a poloidal rotation of the plasma accompanied by a transition with $\mathrm{L}$ to $\mathrm{H}$ mode characteristicis. In IMS the application of a radial electric field has resulted in a poloidal flow that has been directly measured, as discussed in Section II.A. This poloidal rotation is accompanied by changes in the density profile under certain conditions as shown in Figure II.A.4.

In preparation for studies of the currents, a code has been developed which can calculate the effects of the magnetic topology on currents in the presence of islands. An expression for the field error in IMS has been found which gives good agreement with the size and phasing of the islands previously mapped. This field has been used to calculate the effects of the island structure on the current distribution. A probe has beea constructed and tested to measure these currents (and has also been used to measure poloidal ion flow velocity), and an initial scan of the currents along a major radius has been made.

\section{Calculation of the Pfirsch-Schlüter Current}

The Pfirsch-Schlüter current is the parallel current required to keep the divergence of the total current zero, and therefore maintain quasi-neutrality. From the equilibrium equation,

$$
\nabla \mathrm{p}=\underline{\mathrm{J}} \times \underline{\mathrm{B}} ; \alpha \text { is a flux function } \Rightarrow \nabla \mathrm{p}=\mathrm{dp} / \mathrm{d} \alpha \nabla \alpha
$$

one can obtain an equation for the parallel current by letting:

$$
J_{11}=\text { UD dF }
$$


This results in an equation for $\mathrm{H}$ given by:

$$
\underline{B} \cdot \nabla H=-2 /|\underline{B}|^{3}(\underline{B} \times \nabla|\underline{B}|) \cdot \nabla \alpha
$$

$\nabla \alpha$ must be calculated in three dimensional systems to determine the current. A method proposed by Nemov ${ }^{4}$ has been utilized for this purpose by solving $\underline{B} \bullet \nabla \alpha=0$ by means of three coupled ODE's for the components of $\nabla \alpha$ by integration along field lines. This method is attractive for present purposes in that no special symmetries are required and currents in the presence of islands can be calculated. Figure II. $b .1$ shows the geometrical factor $H$ times B (i.e. surpressing only the pressure gradient factor in the current) as calculated for the ideal magnetic configuration of IMS (that is, without magnetic islands). As can be seen, the current is nearly tokamak-like with a $\cos \theta$ dependence, indicative of the large compionent of toroidal curvature to the field structure, and does not vary significantly in going toroidally over a field period.

As an additional test of the code, the factor $H^{*} B$ was calculated for one of the quasi-helically symmetric. (QHS) stellarators discussed further in Section II.C. In these devices, the magnetic field strength, in Boozer coordinates, is nearly helically symmetric (as the name implies) with minimal toroidal curvature effects. Figure II.B.2 shows the resulis of these calculations. The current can clearly be seen to rotate helically through a field period. The direction of the currents reverses over a half field period interval in toroidal angle. This observation is to be expected from the nature of the quasi-helical system and offers a novel possibility of using Pfirsch-Schlüter current measurements as a test to determine if an actual device has achieved QHS.

Field mapping done previously in IMS has revealed the presence of an island located at the $q=3$ surface. A sketch taken off a TV monitor is presented in Figure II.B.3. An ideal $m=3 n=1$ perturbation, with no net toroidal current, was applied to the designed magnetic structure in IMS to model the error field present in the device. The resuit of this perturbation (with the proper phasing and current level) is shown in Figure II.B.4. Reasonable agreement with experiment in island size and location has been attained using this model. [Two main candidates are under investigation as the source of this error: a 'spun' coil, with a poloidal rotation error in its location; and a physical disruption in the current path resulting from a previous internal arc-over between coils several years ago. This topic will be discussed further in the proposed research section.j This error field formulation has been used to calculate the expected current distribution in the presence of the islands in IMS.

Figure II.B.5 shows a plot of the $q=3$ island in the $X-Y$ plane with the value of $H^{*} B$ shown in the $Z$ direction. Below it is the variation of $H^{*} B$ in the poloidal direction for the island seen in the upper-left of the 3-D plot. As can be seen from the Figure, each section of the island looks like a small tokamak, or toroidal system, in the sense that the current reverses in poloidaliy traversing the closed surfaces within the island. 

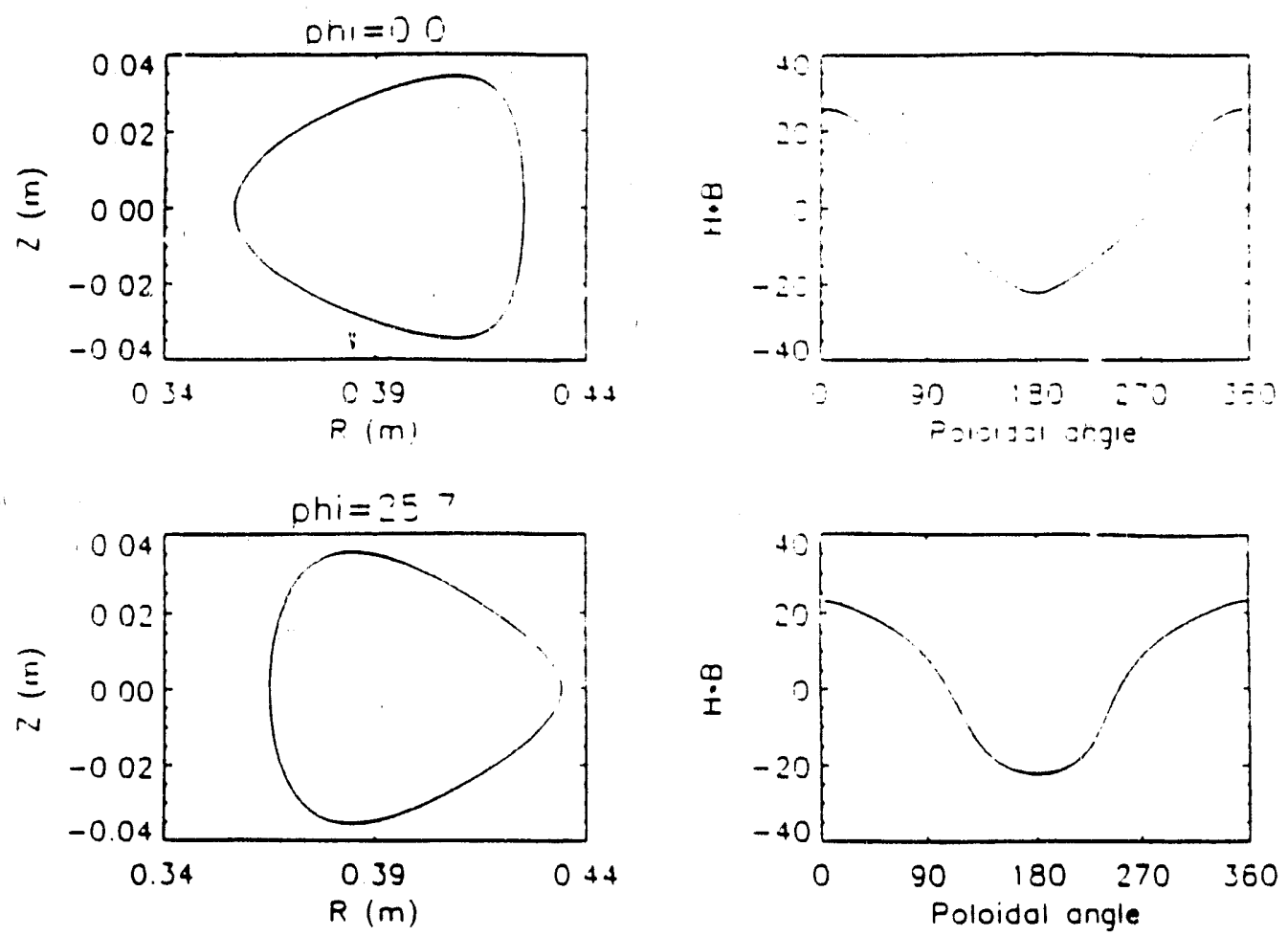

Flux Surfáze and $H^{*} B$ at two toroidal angles in IMS

Figure II.B.1
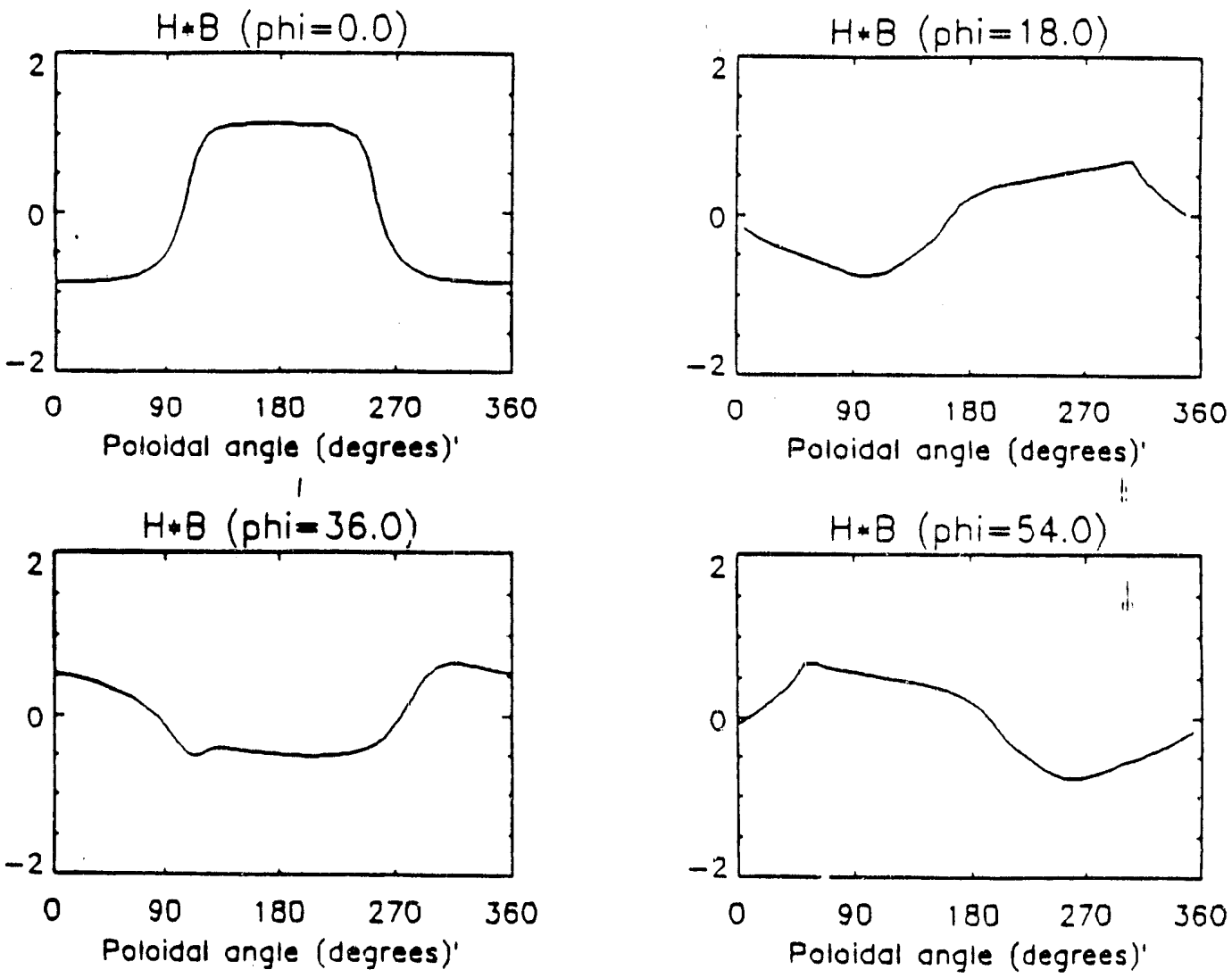

$H^{*} B$ at four toroidal angles within a field period for $Q H S$ system

Figure II.B.2 


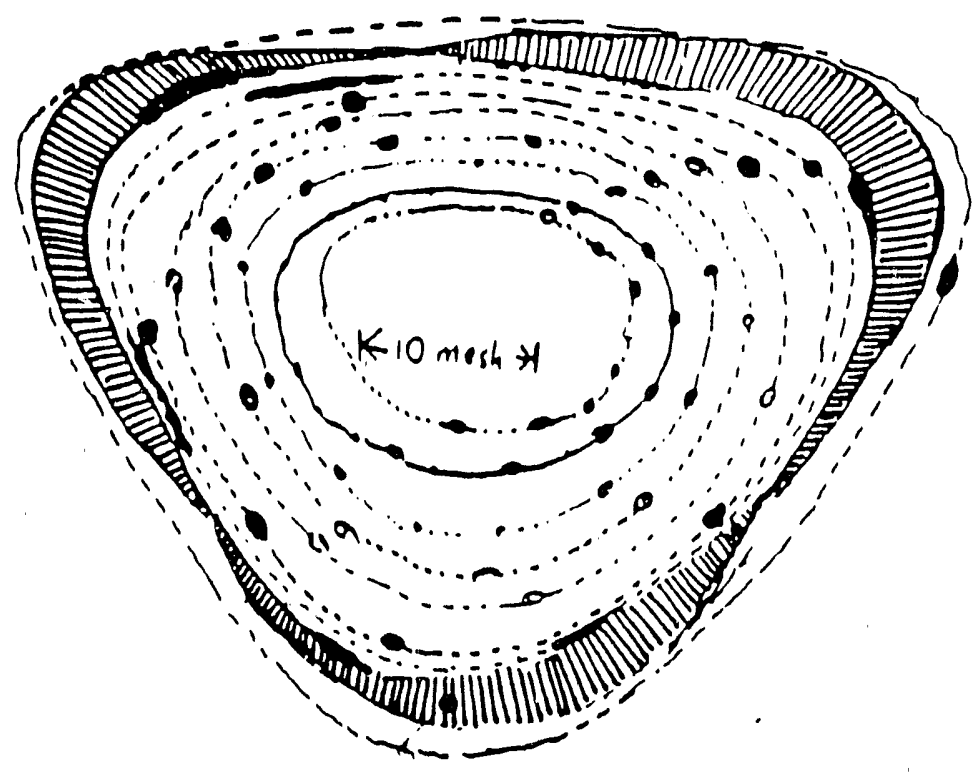

Figure II.B.3

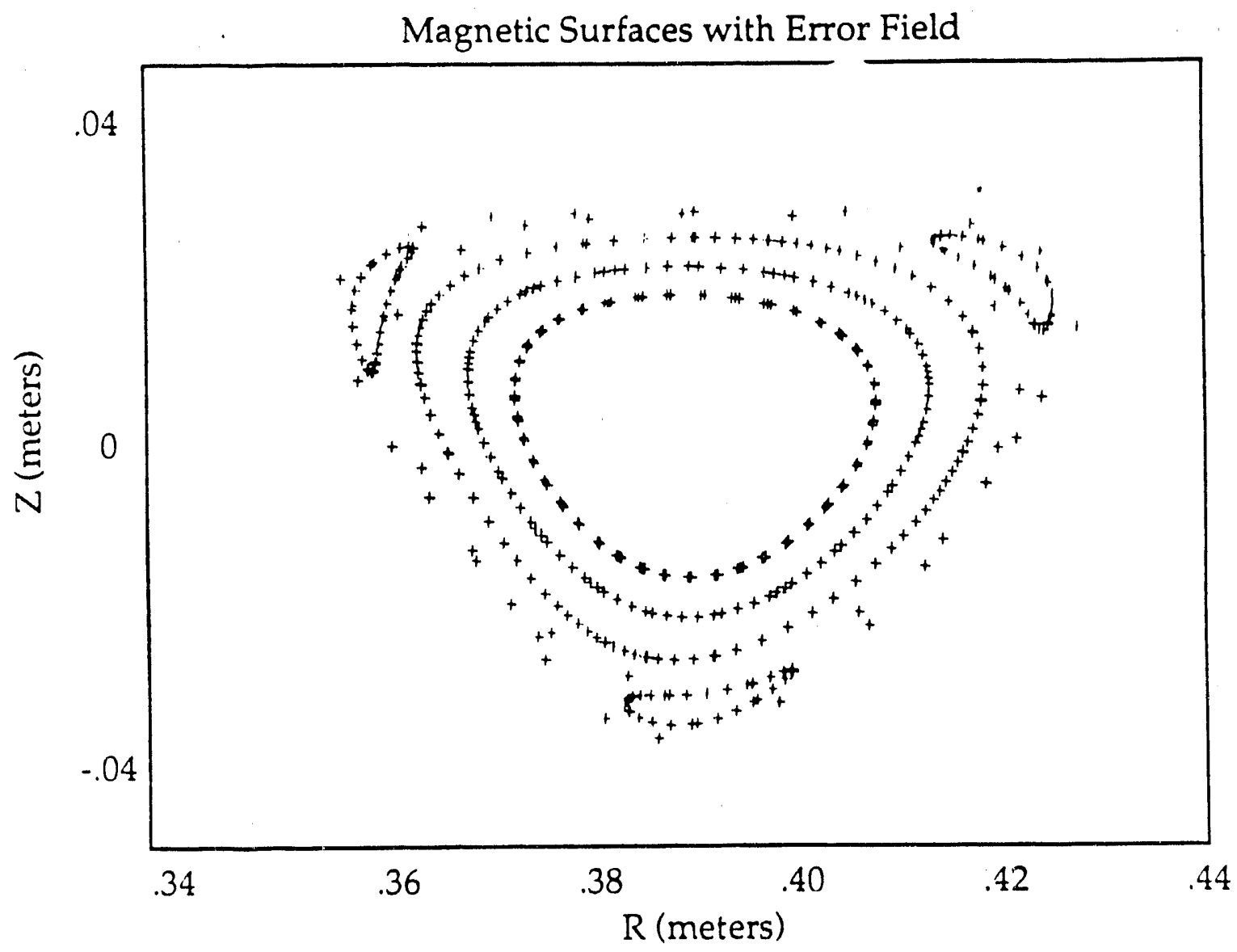

Figure II.B.4 

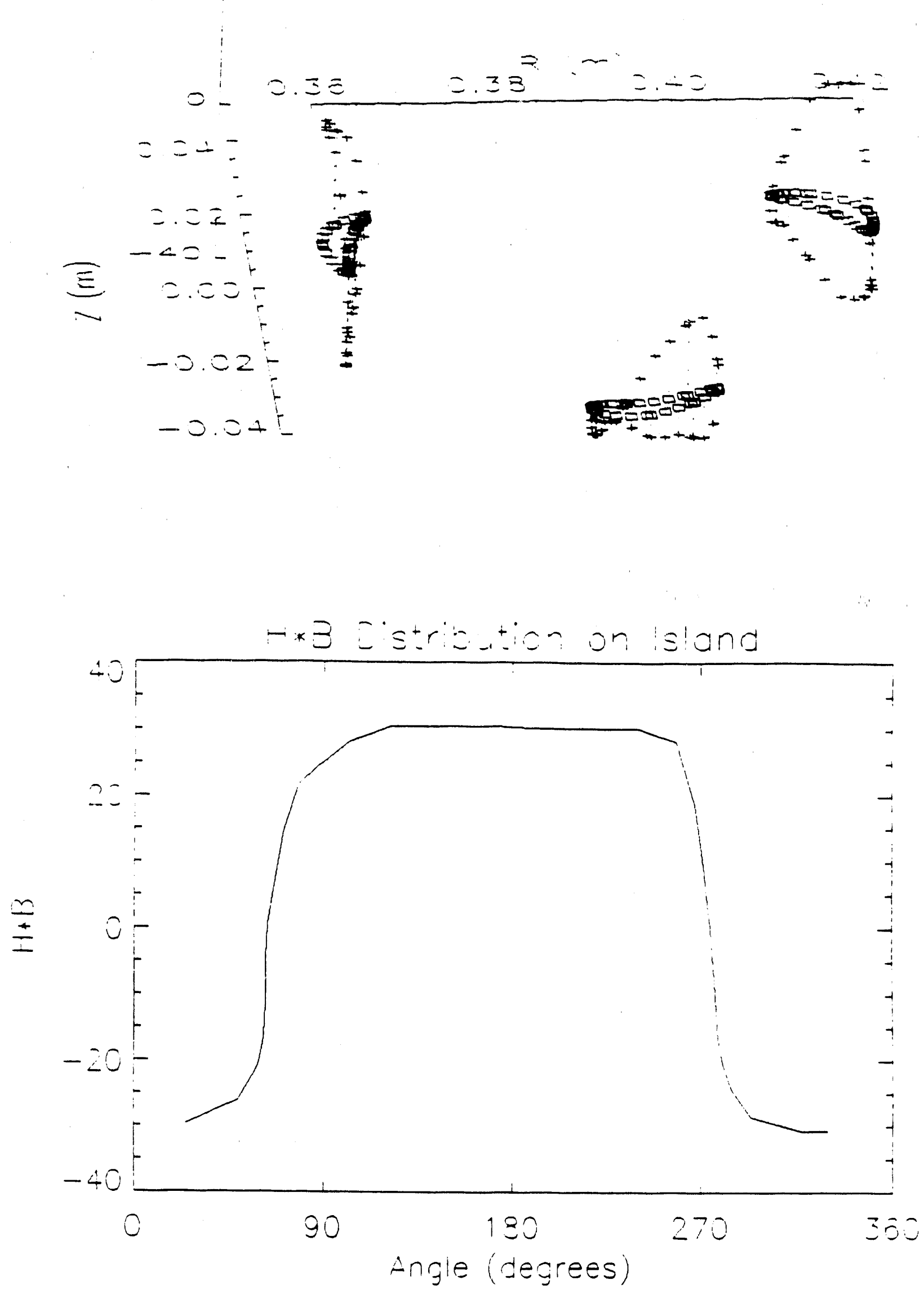

Figure II.B.5 


\section{Current Measurements}

A small paddle probe has been constructed to measure currents in IMS. The probe and its dimensions are shown in Figure II.B.6. The technique relies on orienting the probe faces perpendiclidar to the field lines and measuring the difference in saturation current (ion or electron) to the similarly-biased faces of the probe. With similar thermal currents to the probe faces, the difference is the directed current. Taking two measurements at each probe position, and flipping the probe between shots, obviates the need for precise 'balancing' of the probe at each data location. 'I'he relative standard deviation in the current signals based on 16 consecutive shots is $\sim 10 \%$. The relative standard deviation based on instrumental errors propagated through the calculations is $\sim 15 \%$.

An initial test run of the probe was made by taking radial profiles of density and parallel electron current in $1 \mathrm{~cm}$ steps across the plasma. A measurement of the ion current component showed a similar stucture, but at a level of on'y about $6 \%$ of the electron current, therefore the contribution of the ions to the total current is relatively small. The data from this test run of the probe was compared to calculated currents using electron pressure measurements made simultaneously with the current scan. The pressure was calculated using the measured electron density and by assuming a flat, $5 \mathrm{eV}$ electron temperature profile. Results of the comparison are presented ir. Figure II.B.7. The boxes represent the data poin's of the current as measured in the experiment. The error bar shown on the smooth, theoretical calculated current is due to experimental measurements used in the calculation. The comparison shows qualitative agreement between the measurements and calculations. The effect of the bootstrap current has not been included yet in the calculations. Refined measurements and calculations are in progress and discussed in the Proposed Research.

Large ion flows have been observed with this probe in the case of plasma rotation induced with the biasing electrode. In this case, the probe was oriented to detect poloidally flowing ions. The bias was kept at a fixed potential difference below the floating potential which was monitored by one of the cylindrical probes also located on the paddle probe assembly. The ion 'current', combined with the measured density, gave poloidal flow velocities in good agreement with fluctuation phase delay measurements and $E \times B$ drift velocities from measured potentials. These results are plotted in Figure II.A.7.

\section{References for Section II. B}

1. C. C. Hegna and A. Bhattacharjee, Phys. Fluids B 1(2) (1989) 392.

2. T. Hayashi, T. Sato, A. Takei, Phys. Fluids B 2 (1990) 329.

3. G.R. Tynan, et al., Bull. Am. Phys. Soc. 35(1990)1947.

4. V.V. Nemov, Nucl. Fusion 28 (1988) 1727. 

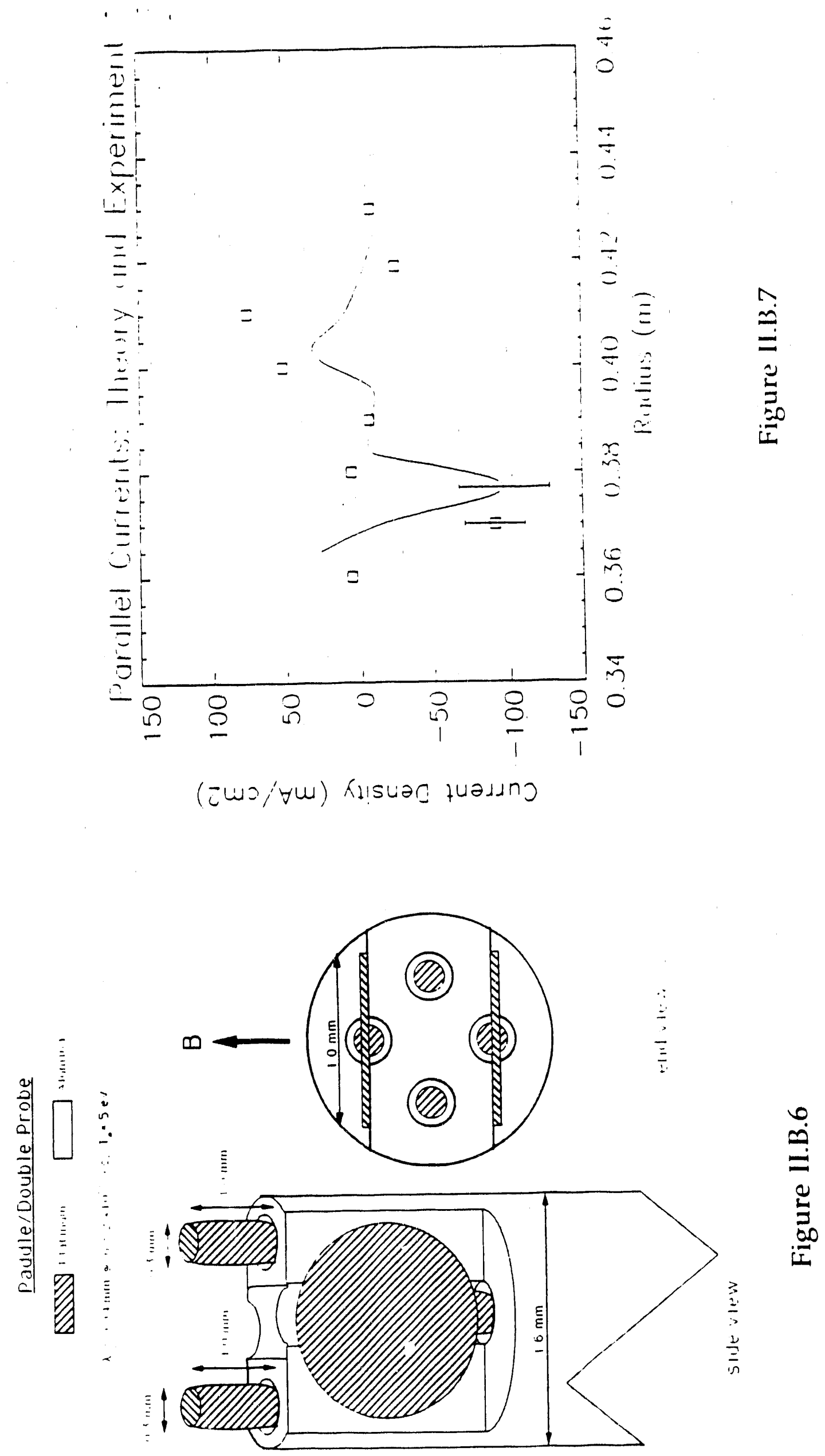


\section{Quasi-Helically Symmetric Configurations}

Quasi-helically' symmetric (QHS) magnetic configurations are an innovative approach to toroidal confinement combining many of the advantages of the tokamak and stellarator concepts. Traditional stellarators suffer from a multitude of different classes of trapped particles due to the interaction of ripples associated with the toroidal nature of thle device and the transform-producing helical winding. These trapped particles lead to increased neoclassical losses for the stellarator $\left(v^{-1}\right.$ regime), among other deleterious effects, as compared to the tokamak with its predominantly toroidal-curvature-driven ripple. QHS configurations, on the other hand, possess a single dominant component in the magnetic field strength analogous to a tokamak. A helical invariant of the motion can be defined for such : system (equivalent to $p_{\phi}$ in a tokamak) ensuring perfect containment of single particles and neoclassical diffusive losses as low as, or lower than, in a similar tokamak. Additionally, these configurations possess all of the other attractive features of the stellarator: inherently steady-state (no current or current-drive needed); no disruptions; external control of magnetic parameters; low recirculating power in a reactor embodiment, etc.

Quasi-helically symmetric systems have been found using the HELIAS 1 design methodology developed at Garching. Early configurations 2,3 had plasma aspect ratios ranging from 11-14. A modest-sized QHS device could contribute directly to the understanding of tokamak transport due to the similarity of the magnetic field structures, without the need for plasma current. At high aspect iatios, however, such a device might be prohibitively large and expensive. Work was undertaken by TSL in collaboration with Garching to investigate lower aspect ratio QHS configurations. The method revolves around non-linear optimization of a set of coefficients describing the plasma boundary (for an equilibrium code) to limit the $|B|$ spectrum to mainly a single component. Symmetry-breaking terms $(m \neq n)$ should be reduced to a level less than $\sim .1 \%$ of the main field strength. Built-in to the optimization process is the requirement for a magnetic well necessary for stabilization of resistive interchange modes, a leading candidate for the cause of anomalous transport in the edge regions of steilarators.

Previous work by the Garching Wendelstein VII-X group had shown QHS configurations could be obtained at aspect ratio of 10 for five-field-period configurations 4 . Attempts to produce QHS configurations with five field periods at lower aspect ratios resulte in deviations from QHS which could not be reduced within the bounds of the o. timization proceedure. Aspect ratios could, however, be reduced significantly for four-field-period configurations. QHS configurations have now been found with aspect ratio 8 , and with increased ( $\sim$ factor of three ) deviations from QHS, at aspect ratio 6 . Three-field-period systems were investigated, but equilibrium convergence problems were encountered even at aspect ratio 8 . 
Figures II.C.1 and 2 shows the flux surfaces computed from the equilibrium code after optimization of the boundary parameters for the four field period, aspect ratio 8 configuration (QHS-48). Figure II.C.1 shows the surfaces at the beginning, $1 / 4$, and $1 / 2$ field period intervals in toroidal angles, while Figure II.C. 2 is a three dimensiv al perspective view of the entire plasma. The helical axis, most easily observed in Figure II.C.1, is necessary to generate a magnetic well (as in the Heliac-type configuration) and to provide the interaction between the helical fields and the toroidal topology to minimize toroidal cdrvature components of the $|\mathrm{B}|$ spectrum in magnetic coordinates.

The deviation of a particles trajectory from a flux surface depends on the variation of the magnetic field strength. Figure II.C.3 shows the $|B|$ on a flux surface at $1 / 2$ the average minor radius for this configuration. True helical symmetry would be manifested by straight $|B|$ lines in this plot. It is clear frcm the plot that only minor deviations from this symmetry exist. The dotted lines in the Figure represent the weak regions of magnetic field strength. The true picture for particle trajectories, of course, resides in the magnetic field strength a particle encounters in moving along a line of force. Figure II.C.4 shows $|\mathrm{B}|$ plotted as a function of toroidal angle in going once around the torus for the surface shown in Figure II.C.3. The ripple along the field line looks virtually identical to that in a tokamak which has. made three poloidal revolutions (i.e. $q=1 / 3$ ). In general, because of the helical symmetry, neoclassical transport analysis of a tokamak carries over directly 5 to QHS configurations with the $q$ value of the tokamak replaced by $1 /(M-1)$, where $M$ is the number of field periods. As a result, $Q H S$ configurations can have reduced neoclassical transport as compared to an equivalent tokamak (here $M=4,1 \sim 1$ > reduction of 3 ).

Figure II.C.5 shows the radial dependence of the components of the $|\mathrm{B}|$ spectrum. From the left-hand side of the Figure, it is clear that the dominant components are the average field strength and fundamental helical component. The right-hand side of the Figure shows an expanded view of the significant components demonstrating the large reduction that has been achieved in the symmetry-breaking terms. In comparison, for it 'conventional' stellarator at aspect ratio 8 , the $\varepsilon_{t}$ related term (toroidal curvature, $n=0, m=1$ ) would have a value of almost .18 , or nearly as large as the $(n=1, m=1)$ m ain helical component.

Optimization studies carried out for the our field period, aspect ratio 6, QHS configuration (QHS-46) were able to reduce s'/mmetry-breaking terms, but not to the extent demonstrated in the QHS-48. Figure II.C.6 and 7 show the $\mid$ B $\mid$ on a flux surface and the radial dependence of ine $|B|$ spectrum respectively. Larger deviations from straight line contuurs are seen in Figure II.C.6 and symmetry-breaking terms are increased by a factor of three from QHS- 48 . Nevertheless, the toroidal curvature at the edge is still reduced to about .3\% in QHS-46, corresponding to a conventional stellarator at aspect ratio $\sim 300$. Further optimization work on this configuration is in progress. 


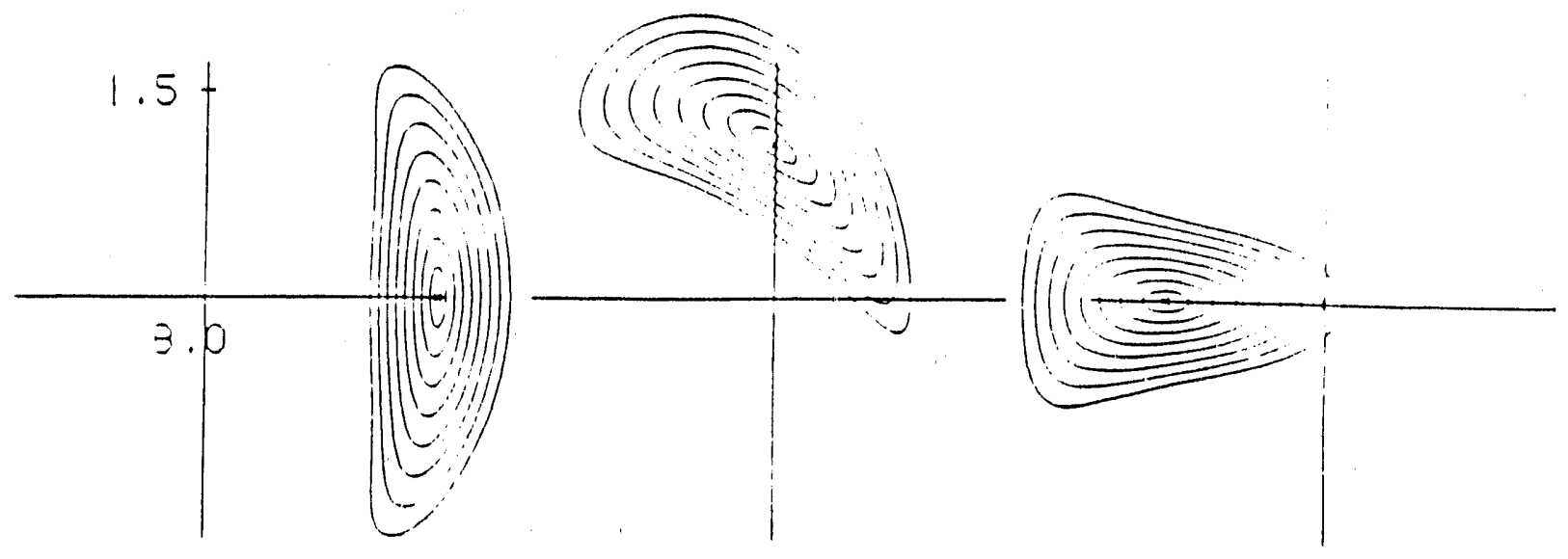

Figure II.C.1

\section{QUASIHELICALLY SYMMETRIC STELLARATOR WITH $N=4$ AND $A=8$}

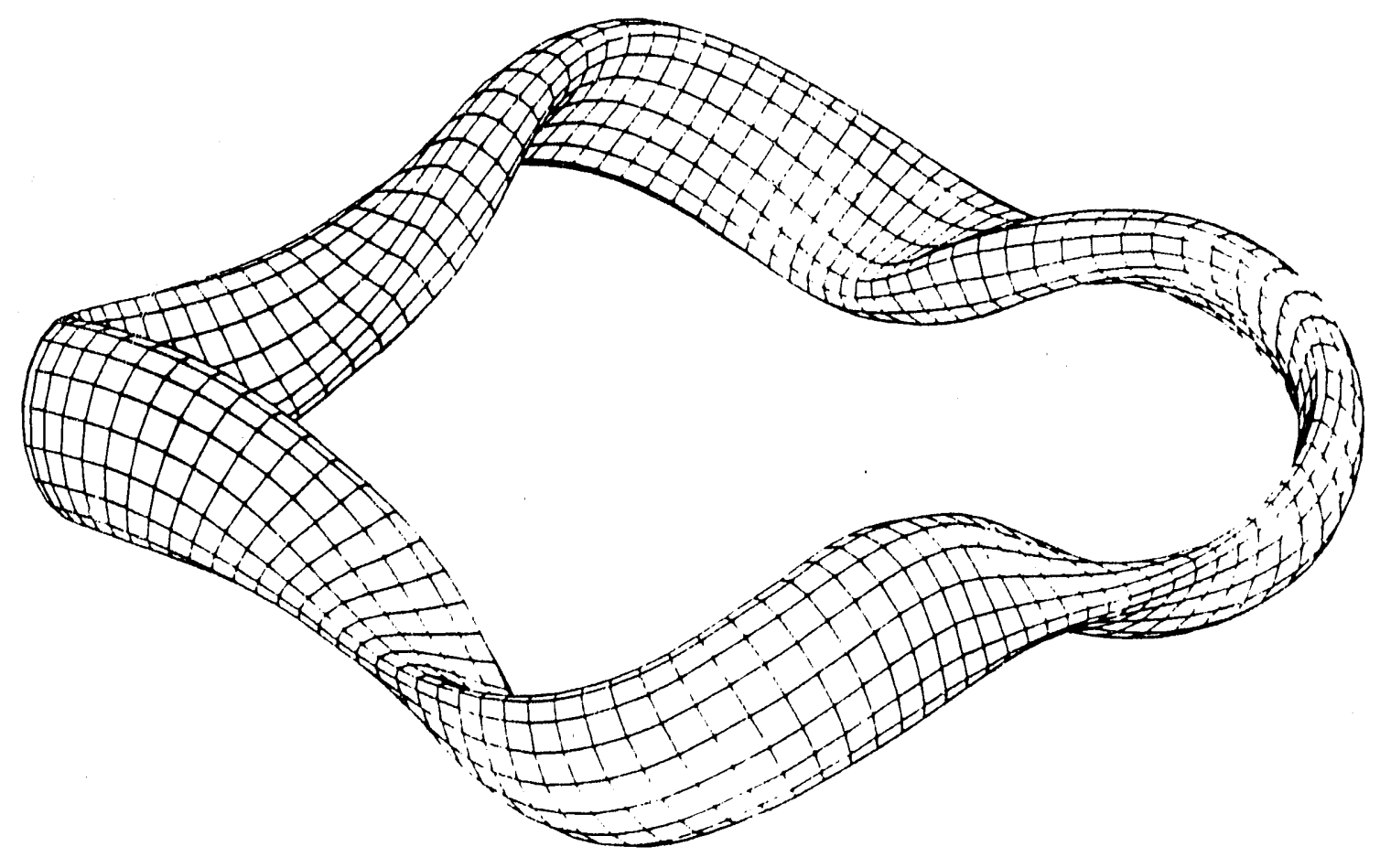

Figure II.C.2 

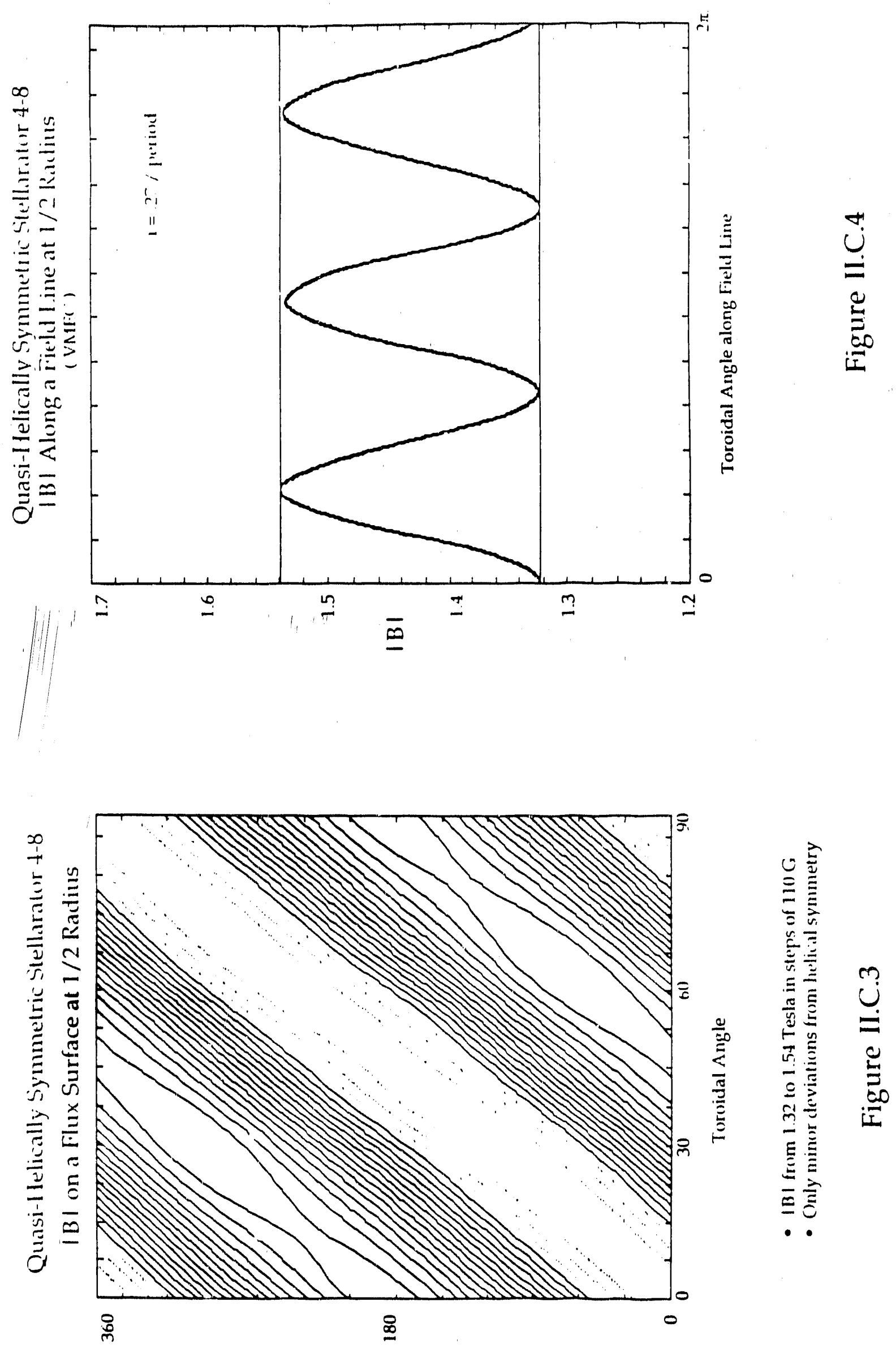


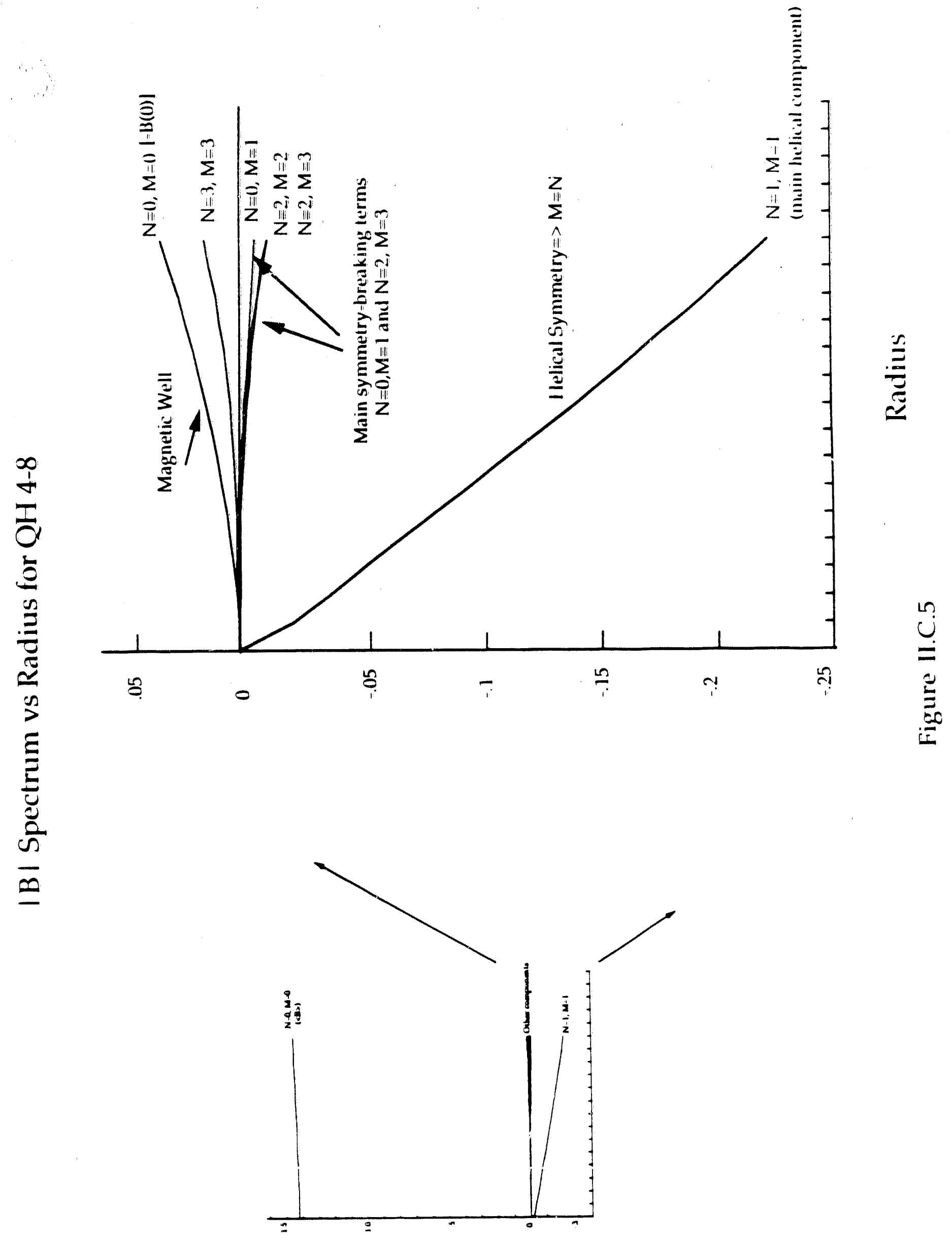



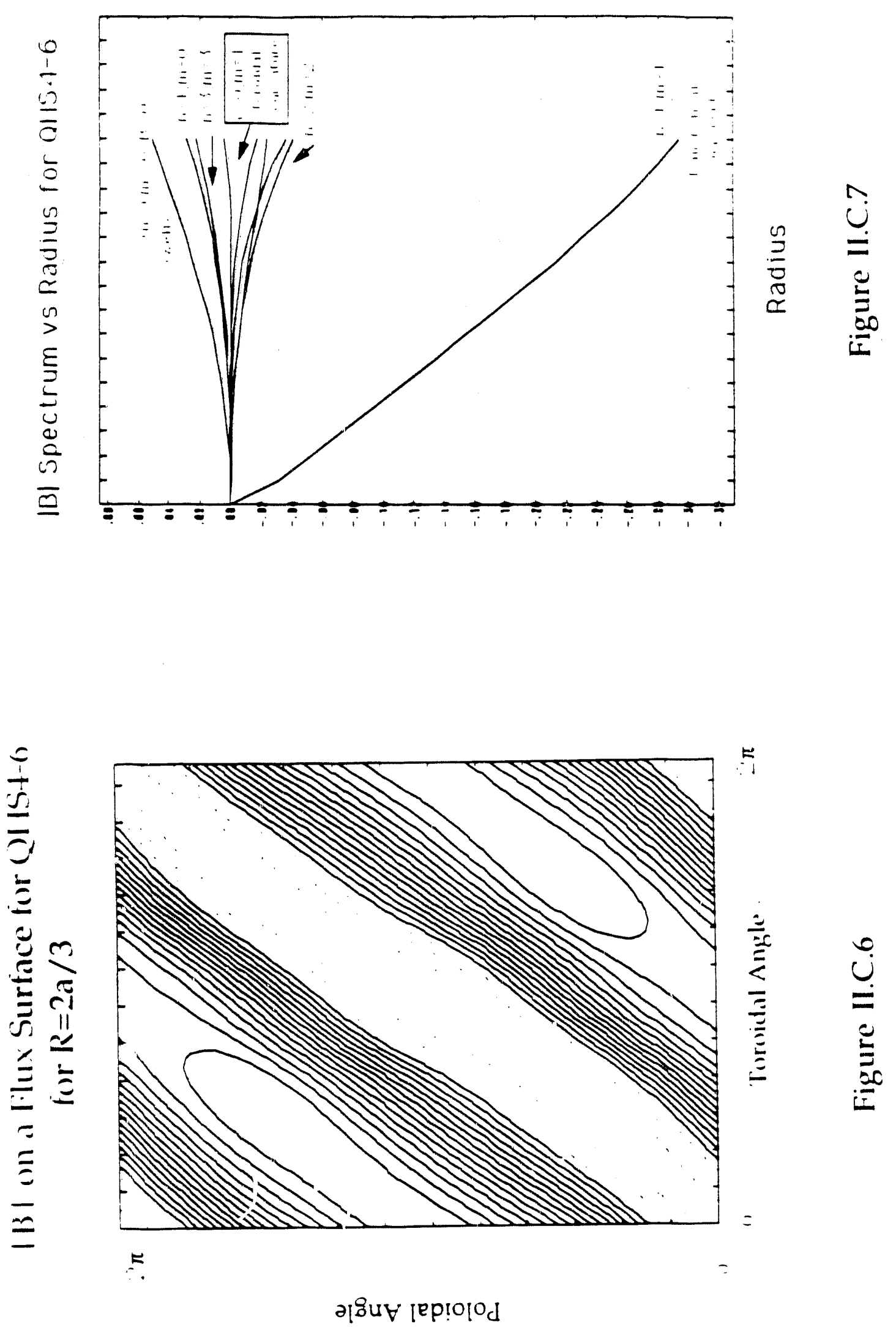
Theories of particle cor tainment in toroidal systems are based on the guiding center drift app oximation; little work has been done on when the drift approximation is justified and how long the drift trajectory represents the true trajectory of a particle. These questions are of particular importance for $\alpha$ particle containment due to their large gyroradius and low collisionality. This topic is presently under anaiytical/numerical investigation by $\mathrm{Dr}$. Allen Boozer with Qun Yao at the College of William and Mary. They are particularly interested in the QHS magnetic structure as it is the only toroidal device which could experimentally test these issues. While theoretical analysis (in the drift approximation framework) for truly helically symmetric systems predicts perfect single-particle confinement (as in a tokamak), the question also arises as to the effects of the slight departure from perfect symmetry present in a QHS system. Boozer and Yao ale currently investigating this issue in collaboration with TSL to determine the degree of QHS necessary for good confinement of $\alpha$-like particles.

As an initial test of single-particle confinement in the QHS-48 configuration, collisionless alpha particles were followed in reactor-sized QHS systems by Lotz at Garching6. One hundred trapped particles were launched from an internal flux surface (corresponding to an effective birth location of alpha particles) and followed until lost or reaching a thermalization time ( .1 seconds). Figure II.C. 8 plots the fraction lost as a function of time for a reference $1=2$ stellarator device. All of the particles are lost before even $.1 \%$ of the thermalization time required is reached. This is in contrast to the results of the simulation applied to the QHS-48 configuration shown in Figure II.C. 9. Here, only one particle out of the original 100 failed to be confined for the required thermalization time. It is clear from this calcuiation that single-particle trajectories are not seriously degraded in QHS-48 due to deviations from perfect symmetry. An analysis for QHS- 46 has not yet been done to determine the effects of the increased deviation from QHS (as compared to QHS-48) on particle contairument.

Single particles in this simulation were globally well contained. A Monte-Carlo calculation of the diffusion coefficient for the QHS-48 configuration was performed using a code 7 developed by a TSL graduate student (while working at $\mathrm{CHS}$ in Nagoya) and results compared to analytic calculations for an equivalent tokamak. The results are shown in Figure II.C.10 where it is clear that: the $1 / \mathrm{v}$ regime has disappeared for the QHS device; the diffusion coefficient has a similar collisionality dependence to the tokamak, and; magnitudes of the diffusion coefficient are reduced by approximately the factor of three predicted by analytic theories.

\section{References for Section II.C}

1. Nührenberg, J. and Zille, R., Phys.Lett. A 114 (1986) 129.

2. Nührenberg, J. and Zille, R., Phys.Lett. A 129 (1988) 113.

3. Nührenberg, Presented First Intl. Toki Conf., Toki Japan (1989).

4. See Proceedings of 2 nd IVorkshop of Wendelstein VII-X, EUR-11705 EN (1988).

5. Boozer, A.H., Phys. Fluids 26, 496 (1983).

6. W. Lotz, private communication.

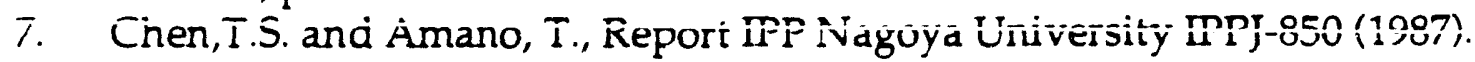


' ${ }^{\prime}$ of Lost Trapped $\alpha$ Particles
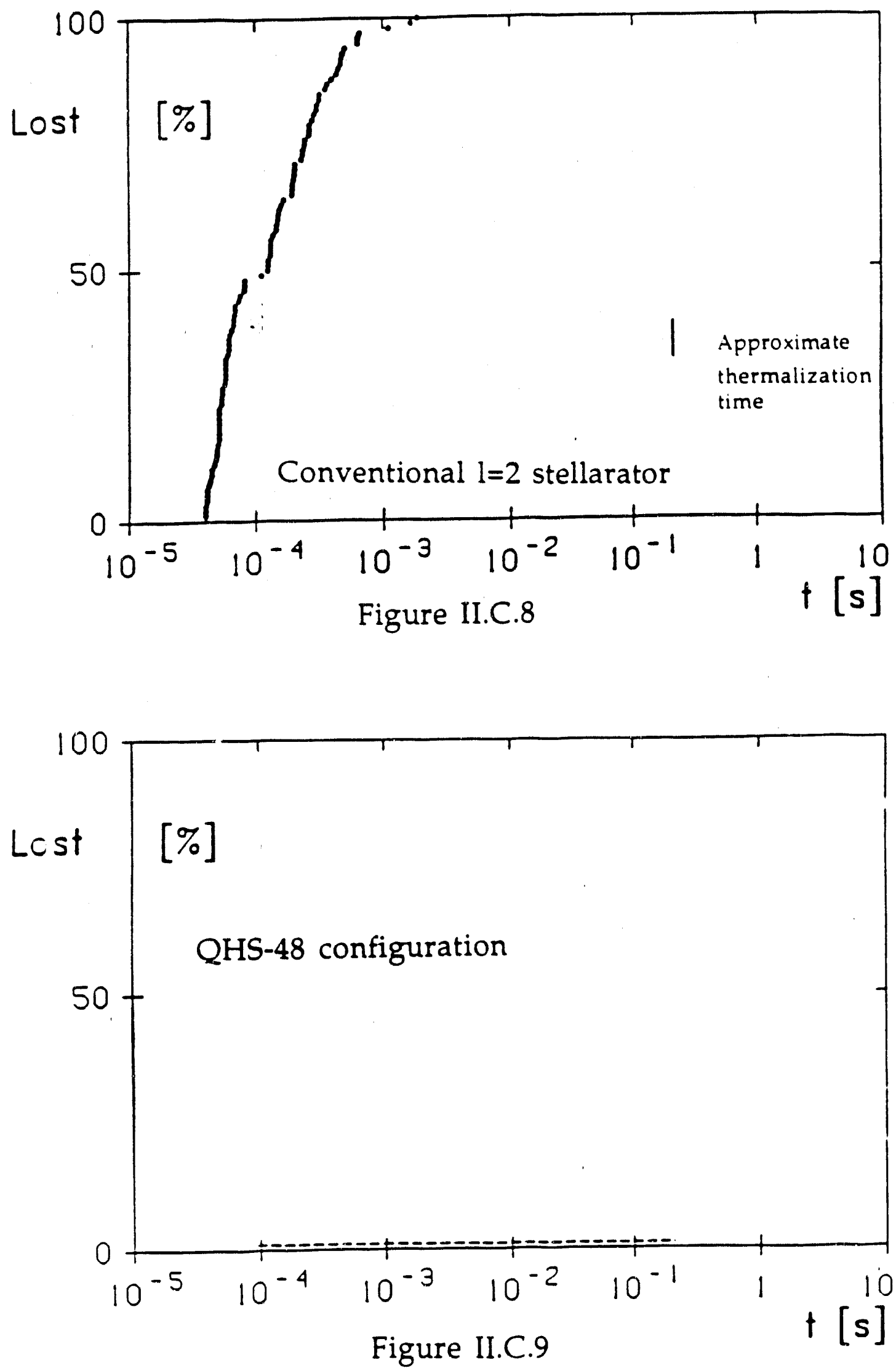


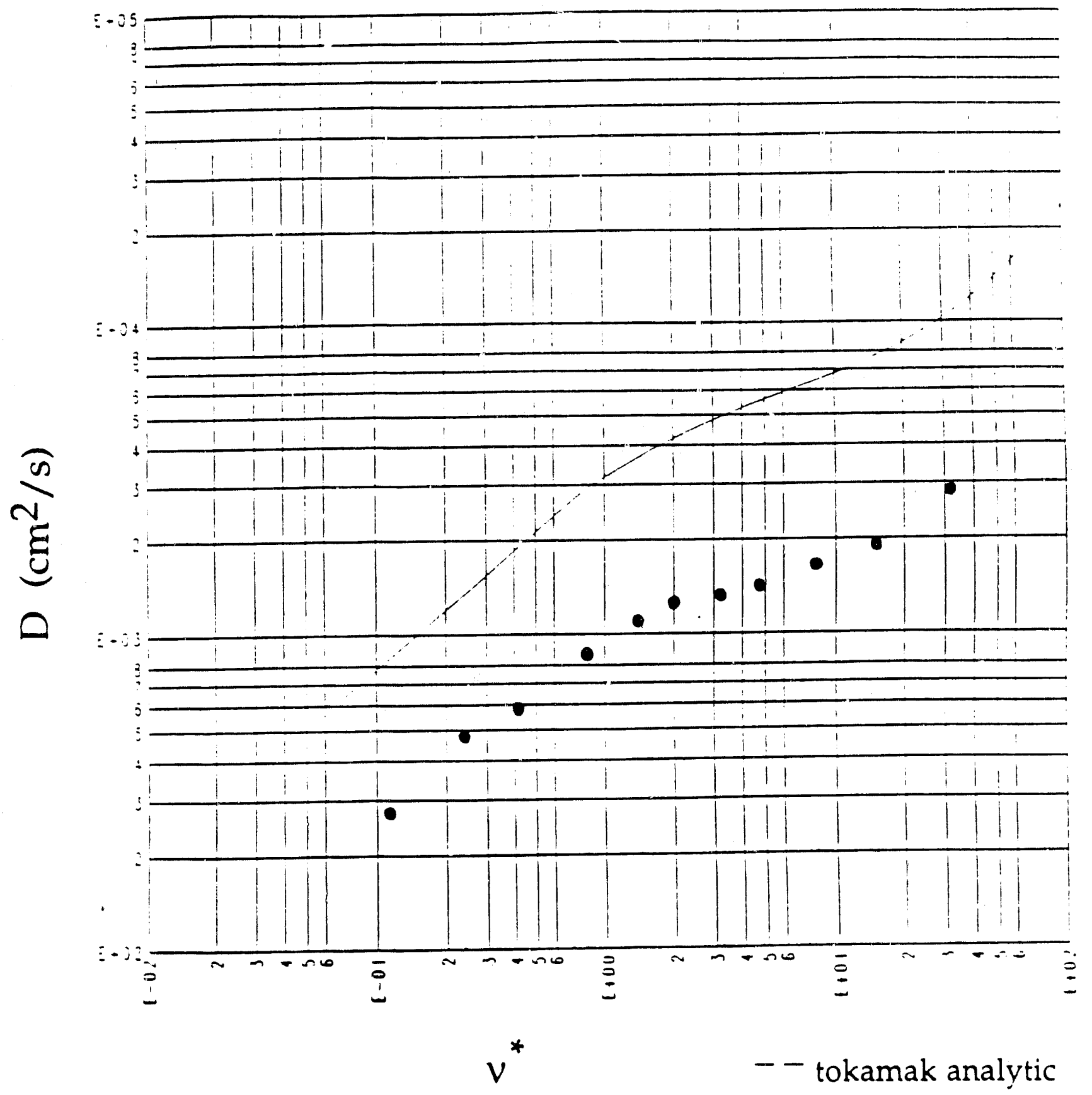

- Monte-Carlo QHS-48

Figure II.C.10 


\section{Proposed Research}

Work during the present grant period has put the Torsatron/Stellarator laboratory in a position to examine some important issues of the physics of toroidal transport. Proposed research for the next two fiscal years will build upon this base by examining electric fields, plasma spin-up and poloidal rotation and fluctuations of the potential, density and temperature. Flow structures in the presence of magnetic islands and their effects on poloidal asymmetries, as well as two-dimensional plasma current distributions near the island will be examined (prior to obtaining a non-islated configuration in IMS). Plasma potential structures driven by direct orbit loss associated with ECRH will be investigated using discriminator screens (on divertor strike-plates) and gridded energy analyzers installed during field error repair. Innovative toroidal configurations will be assessed to ascertain their possible role in advancing the understanding of tokamak transport.

\section{A. Fluctuations and Edge Transport}

Fluctuation-induced transport, plasma rotation and viscosity, and the effects of magnetic field errors are crucial issues for general toroidal confinement. Recent tokamak research, particularly in CCT and DIII-D, indicate that during the L-H transition the plasma poloidal rotation and radial electric field increase, the fluctuation-induced transport decreases, and confinement improves. However, there is uncertainty over the interpretation of certain experimental measurements and the validity of theoretical models put forth to explain tokamak transport. The demonstrated abilities to make detailed measurements of space potential throughout the plasma using emissive probes and to alter radial electric fields using biasing electrodes, in MMS, will be exploited to examine some asperts of these issues.

Experimental activity will concentrate on measurements of the potential and density fluctuations, and associated transport, near the locations of induced plasma poloidal rotation in IMS. Fluctuations have been reduced in this region of the plasma in IMS, although increased in other regions. Preliminary results show that we are able to control the strength of the introduced radial electric field in IMS by varying the applied bias to the electrode. Radial electric field strength and distribution will be measured as a function of applied bias and correlated with poloidal rotation measurements. This data will then be used to examine the changes in fluctuation levels and characteristics as a function of the poloidal flow velocity. 
Another issue of uncertainty in tokamak transport is the cause of the spin-up associated with the L-H transition. Experimentally we will address two of the theoretical models proposed to explain the poloidal flow generation and hopefully obtain some insight into the side issue of the experimental absence (to date) of $\mathrm{L}-\mathrm{H}$ transitions in stellarators.

One model of poloidal flow generation in a tokamak has been proposed by Shaing who found that the balance between the $J \times B$ force due to direct orbit loss at the tokamak edge and the poloidal viscosity can yield multiple solutions, depending on collisionality, for the poloidal flow. By measuring the time-dependent spin-up of the plasma, under different levels of biasing, we can obtain an estimate of the poloidal viscosity for IMS. This will be compared to calculations based upon theoretical models, developed in the last ten years, of the viscosity in a stellarator by Shaing, and also by Coronado. Dr. Coronado is spending his sabbatical from the University of Mexico at the TSL for one year, and will assist in these calculations.

An alternative explanation of poloidal flow generation in a tokamak has been proposed by Diamond and Kim. In their model, the poloidal flow is generated by the turbulence, more specifically, the gradient of the Reynolds stress in the radial direction. Experimentally, this means measuring the fluctuations in the radial and poloidal electric fields as a function of radius. A probe to measure plasma (space) potential fluctuations in three dimensions has already been built and implemented on IMS. A comparison of flows predicted from the measured gradient of the Reynolds stress to the actual measured poloidal flow will be made. Small poloidal flows are observed in IMS in the absence of electrode biasing. These flows reverse direction and increase in magnitude with the application of bias. Fluctuation levels and characteristics under these two conditions will be compared.

B. Plasma Currents and Flows in the Presence of Magnetic Islands

In CCT, 2-D measurements of density and potential indicate that $m=3$ or 4 poloidal asymmetries at the $q=3$ surface exist in the form of 'islands' which disappear after the transition to the $\mathrm{H}$ mode. It is unclear whether the asymmetries observed in CCT are convective cells or a result of field errors. If the asymmetries are due to field errors, the question arises as to whether this is something intrinsic to only CCT, or perhaps a feature of larger tokamaks as well. The question takes on added significance in the light of recent work by Reiman ( and earlier work by Boozer) which indicates that tokamaks may have a greater sensitivity to field errors than stellarators because of the interaction of the error fields with the ohmic current. 
In IMS, we have already measured $m=3$ asymmetries located at the $q=3$ surface. However, in contrast to $C C T$, we have also measured the vacuum magnetic surfaces and know that there is an island at this location. We have also been able to induce a large poloidal flow and radial electric field in the plasma by extracting electrons with a biased electrode. Experiments in the near-term will utilize the island present in IMS by measuring the two dimensional density and potential profiles, under various bias conditions and poloidal flow velocities, to see whether the rotation reduces the asymmetries associated with island structures.

Recent independent calculations by Cary, Hegna, and Hayashi indicate that equilibrium beta limits in stellarators may be determined, not by the Shafranov shift, but rather the overlap of magnetic islands induced by the Pfirsch-Schlüter current. We will measure the current and pressure profiles in two-dimensions, and will compare measured currents to theoretical calculations. Full two dimensional scans permit examination of the Pfirsch-Schlüter currents both in regions of well-formed magnetic surfaces (interior) and, prior to removal of the IMS field error, in islated configurations. Concurrently, a dipole coil will be used to experimentally investigate the source of the island-producing error.

\section{Plasma Potential Structures and Direct Orbit Loss with ECRH}

Direct-loss orbits in stellarators can drive potential variations on magnetic surfaces and radial electric fields. The perpendicular currents associated with this loss could impart a J $\times$ B torque to the plasma as well. Little work has been done to date, however, on the effects of electron-cyclotron resonance (ECR) heating on poloidal potentials and radial electric fields, and the resulting transport, especially in stellarators.

The difficulty with understanding direct orbit losses from an experimental perspective is that in many devices there is no way to measure the spatial distribution of the direct loss. However, because of the 63 diveriur strike-plates located in the divertor regions of IMS it is possible to obtain the spatial distribution of the particle losses in our device. Preliminary experiments have shown that, for the most part, the ion and electron flows are consistent with the grad-B drift direction for each species. Also the flows are, to within $20 \%$, equal to each other which is at least a step towards confirmation of ambipolarity.

A comprehensive set of gridded-energy analyzers and discriminator screens (for the divertor strike-plates) will be added to IMS during the downtime needed for correction of the field error. Measurements of the radial and poloidal electric fields will be correlated to loss currents (associated with electron-cyclotron heating) measured through these additional diagnostics to understand whether the asymmetric flow is responsible for the creation of the ambipolar electric field and potential variations on magnetic surfaces. 
Variations in the direct loss rate can be achieved by alteration of the helical ripple profile (through use of the vertical field), variation of the resonance location, and through increased ECRH power (now available using the source on loan from the Phaedrus group). By comparing the energy distribution of the particles at divertor locations with high electron or ion fluxes to measurements at other locations, one can determine whether the particles which constitute the asymmetric flow belong to a class of particles different from the bulk of the plasma. If there is a correlation between the radial electric field and the loss-cone orbits, then one would like to examine whether there are any changes in the fluctuation-induced transport as a function of loss rate. To this end, we would make detailed measurements of the density and potential fluctuations under these conditions (as discussed in Section III.A).

\section{Innovative Toroidal Systems Studies}

The quasi-helically symmetric class of stellarators offers expanded possibilities for stellarator contributions to the understanding of toroidal transport due to the primarily single-harmonic $|\mathrm{B}|$ spectrum along field lines. QHS configurations have now been found at aspect ratios commensurate with present-day stellarator experiments, implying that a physical device could be made on a moderate scale.

Experiments on ATF and Heliotron $E$ have observed increasing electron thermal conduction losses near the edge, in contrast to neoclassical predictions. Both devices have a magnetic hill in the outer regions of the plasma. Resistive interchange turbulence, which is not stabilized by shear, gives the proper scaling for the thermal conduction, with an increasing $\chi_{e}$ toward the edge. Resistive interchange modes are thus a possible candidate for anomalous transport in the edge regions of stellarators, analogous to the possible role of resistive ballooning in tokamak edges. QHS configurations have been determined with a side constraint on the optimization proceedure that a magnetic urell extends into the edge regions (stabilizing resistive interchange). As a result, QHS configurations should have transport closer to neoclassical if resistive interchange turbulence is the cause of stellarator anomalous transport. The change from good to bad curvature over a field period in QHS fields implies reduced connection lengths for ballooning modes as well. Parallel currents have been discussed by Wobig as a possible source of energy for driving many possible mechanisms of anomalous transport. While not initially optimized for reduced parallel currents, the QHS-48 configuration discussed in Section II.C has $\left\langle\left.\mathrm{J}_{1}\right|^{2} / \mathrm{J}_{\perp} 2\right\rangle$ on the order of unity. The simplified ripple structure in a QHS configuration also results in reduced parallel viscosity as compared to conventional stellarators. We will theoretically calculate the expected poloidal viscosity in QHS systems in collaboration with Dr. Coronado. While an L-to- $\mathrm{H}$ mode transition has not been observed to date in a stellarator, the similarity of $|\mathrm{B}|$ along the field in a QHS to a tokamak might make the transition possible in such a configuration. An interesting variation to quasi-helically symmetric configurations is the quasi-axi-symmetric (QAS) field. In this case the $|\mathrm{B}|$ spectrum 
is optimized so that the $(m=1, n=0)$ mode is the dominant component, as in a tokamak, with no need for current or current drive. We will continue examination of the QHS/QAS concepts to determine the possible benefits and practicality of such systems as applied to understanding anomalous toroidal transport.

The drift approximation and guiding-center orbits are fundamental tools for estimating alpha particle confinement and heating. There are questions as to the validity of these calculations for the large gyroradii, nearly collisionless long-time behavior characteristic of alphas. The QHS/QAS systems offer the only possible experimental test of the drift approximation and single particle confinement in a torus. This attribute is due to the existence of an invariant of the motion and the lack of ohmic current (required to produce the confining fields in tokamaks and RFP's). Collaborative work will continue with Dr. Allen Boozer and Qun Yao at the College of William and Mary to identify the degree of symmetry needed to make a meaningful test of the drift approximation and the general effects of deviations from QHS/QAS on particle confinement.

A very high degree of quasi-helical symmetry has been obtained for the QHS-48. We will continue to try to reduce variations from QHS in the four field period aspect ratio six case, as well as investigate four field periods at aspect ratio seven. Coil configurations which produce the required magnetic geometry have been found and will be optimized further to reduce coil curvature and maintain plasma-coil distances within acceptable windows. The sensitivity of magnetic surface shape and $|B|$ spectrum to coil winding harmonics will be studied to understand accuracies to which coils would need to be built and limitations they would necessarily introduce into a physical system. 
E. Projected Goals, Schedule and Capital Equipment Request Experimental Plan

\section{Activity} 1. Determine radial electric field st:ength and distribution as a
function of bias voltage/current.

Expected

Comp.

Date

$12 / 91$

$2 / 92$

$3 / 92$

$4 / 92$

$6 / 92$

$8 / 92$

$9 / 92$

8. Take the machine down for repair and diagnostic installation

9. IMS operational; remap vacuum surfaces; extemal island
induction/control

10. Comparison of pressure/potential asymmetries with island
existence/widths. $6 / 93$

:1. Correlation of radial electric fields with direct loss orbits 

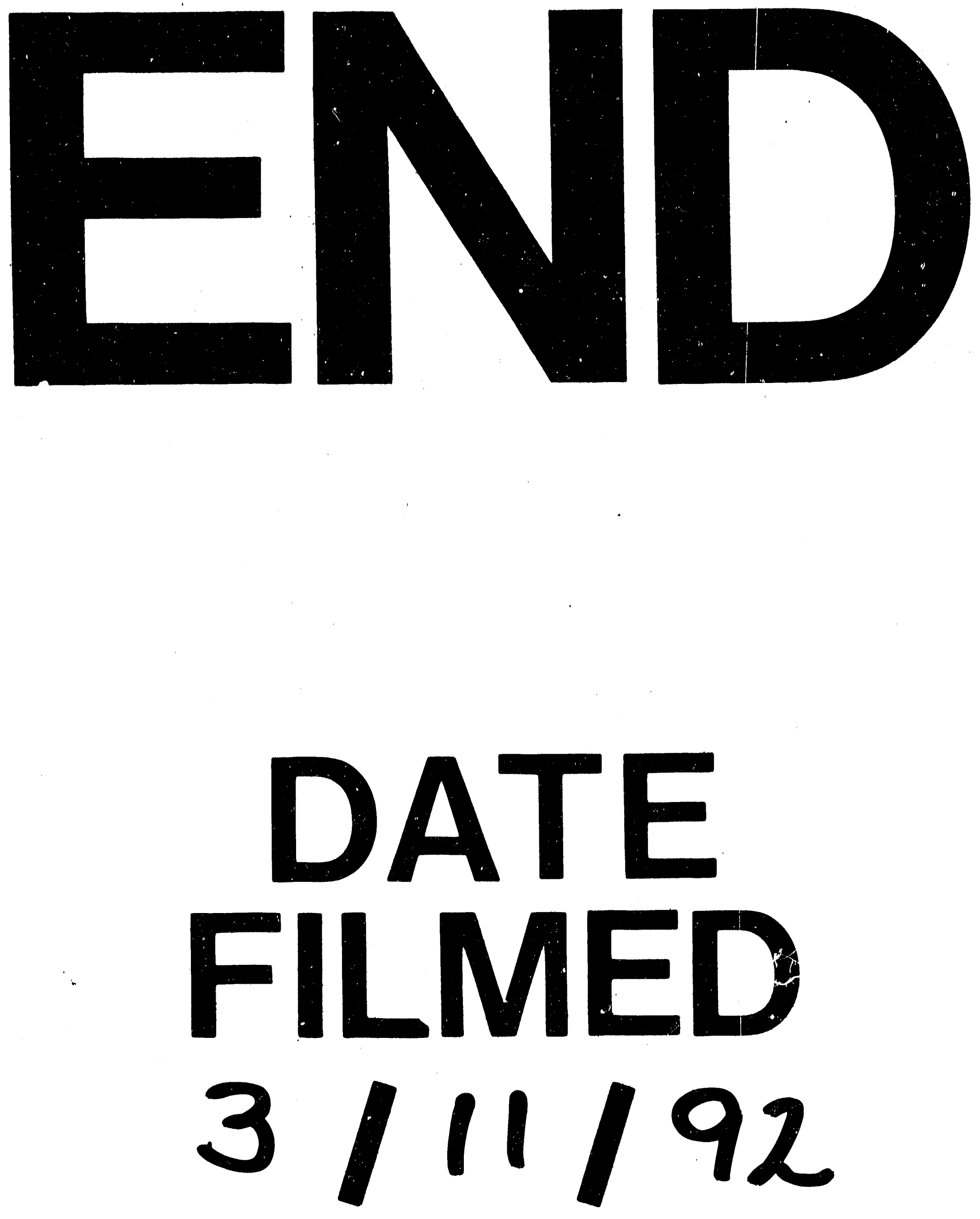

I 
